TRANSACTIONS OF THE

AMERICAN MATHEMATICAL SOCIETY

Volume 364, Number 7, July 2012, Pages 3751-3779

S 0002-9947(2012)05544-9

Article electronically published on February 17, 2012

\title{
CHARACTERISTIC CLASSES AND EXISTENCE OF SINGULAR MAPS
}

\author{
BOLDIZSÁR KALMÁR AND TAMÁS TERPAI
}

\begin{abstract}
The existence of a corank one map of negative codimension puts strong restrictions on the topology of the source manifold. It implies many vanishing theorems on characteristic classes and often even vanishing of the cobordism class of the source manifold. Most of our results lie deeper than just vanishing of Thom polynomials of the higher singularities. We blow up the singular map along the singular set and then perturb the arising nongeneric corank one map.
\end{abstract}

\section{INTRODUCTION}

We work in the smooth category. Suppose an $n$-dimensional manifold $M$ admits a Morin map $f$ into an $(n-k)$-dimensional manifold $Q^{n-k}$, where $k$ is odd. First, we blow up $M$ along the singular set $\Sigma$ of $f$ and then denote the resulting manifold by $\mathrm{Bl} M$. Blowing up is a natural geometric construction which relates the tangent bundles $T \mathrm{Bl} M, T M$ and $T \Sigma$ and the normal bundle of $\Sigma$ to each other in a controllable way. Then we give a concrete perturbation of the composition $f \circ \pi$, where $\pi: \mathrm{Bl} M \rightarrow M$ is the blowup projection. The differential of this perturbation gives us a fiberwise epimorphism $T \mathrm{Bl} M \oplus \varepsilon^{2} \rightarrow T Q$, thus the formal difference bundle $T \mathrm{Bl} M-\pi^{*} f^{*} T Q$ is stably equivalent to a $(k+2)$-dimensional bundle 1 Hence its Stiefel-Whitney characteristic classes $w_{r}$ vanish if $r>k+2$. Thus by the blowup construction we obtain relations between the characteristic classes and numbers of $T M$. As we show, these relations are not the consequence of the vanishing of the Thom polynomials of the forbidden higher corank singularities. One of our main results is that most manifolds admitting Morin maps into stably parallelizable manifolds are null-cobordant. Our results are of the following type.

Received by the editors June 7, 2010 and, in revised form, December 15, 2010 and January 7, 2011.

2010 Mathematics Subject Classification. Primary 57R45; Secondary 57R75, 57R25, 57R20.

Key words and phrases. Singularity, Morin map, fold map, blowup, Morse-Bott map, cobordism, Dold relations, geometric dimension.

The first author was partially supported by the Magyary Zoltán Postdoctoral Fellowship and OTKA grant NK81203.

The second author was supported by OTKA grant NK81203.

${ }^{1}$ Suppose $Q$ is stably parallelizble; then $T \mathrm{Bl} M \oplus \varepsilon^{1}$ has $n-k-1$ linearly independent sections. Denote by $\mathcal{P}_{n-k-1}$ the space of smooth closed connected $n$-dimensional manifolds $X$ with the property that $T X \oplus \varepsilon^{1}$ has $n-k-1$ linearly independent sections. By using the terminology and notation of Mi97, if a manifold in the metric space $\operatorname{Diff}(\mathrm{n})$ admits a Morin map into $Q$, then its distance from $\mathcal{P}_{n-k-1}$ is at most one. 


\subsection{Relations among characteristic classes.}

Theorem 1.1. Let $M$ be an n-dimensional orientable, closed manifold admitting a Morin map $f: M^{n} \rightarrow S^{n-k}$ into the $(n-k)$-sphere $S^{n-k}$, where $k$ is odd. Then for any pair of multiindices $R=\left(r_{1}, \ldots, r_{m}\right)$ and $S=\left(s_{1}, \ldots, s_{m}\right)$ of (any) equal length $m$ and (any) equal degree $\sum_{j=1}^{m} r_{j}=\sum_{j=1}^{m} s_{j}$, we have

$$
w_{R}(M)=w_{S}(M)
$$

if all the entries $r_{j}$ and $s_{j}$ are at least $k+3$. When $M$ admits even a fold map, then (1.1) holds even for entries at least $k+2$.

Remark 1.2. (1) Actually we prove a slightly stronger version of the above theorem replacing $S^{n-k}$ by any orientable $(n-k)$-dimensional manifold $Q$ such that $w_{i}(Q)=0$ for $i>K$. In this version (1.1) holds if $r_{j}$ and $s_{j}$ are at least $k+3+K$ (resp. $k+2+K)$.

(2) Theorem 1.1 holds also if $M$ and $Q$ are possibly nonorientable and the Morin map of $M$ into $Q$ is a cusp map.

(3) If $k \geq 0$ and $k$ is even, then (1.1) obviously holds for a fold map if $r_{j}, s_{j} \geq k+2$ since $w_{j}(M)=0$ for $j \geq k+2$; see An04, Sae92.

(4) By Ron72] the $\mathbb{Z}_{2}$-Thom polynomial for the $\Sigma^{l+2}$ singularity is $w_{l+2}^{2}+w_{l+1} w_{l+3}$ (with the normal Stiefel-Whitney classes). Let $1 \leq m<2^{n-1}$ and ask about the existence of a Morin map $f: \mathbb{R} P^{2^{n}-m} \rightarrow \mathbb{R}^{2^{n}-m-k}$, where $k+1 \geq m$. The total normal Stiefel-Whitney class $\bar{w}\left(\mathbb{R} P^{2^{n}-m}\right)=(1+a)^{m-1}$, where $a$ denotes the generator of the cohomology ring with $\mathbb{Z}_{2}$ coefficients of $\mathbb{R} P^{2^{n}-m}$. Hence all the Thom polynomials $w_{l+2}^{2}+w_{l+1} w_{l+3}$ are zero if $l+2 \geq m$. This means that the Thom polynomials for the $\Sigma^{l+2}$ singularities cannot give obstructions for the existence of a Morin map $f$ if $l+2 \geq m$. In particular, if $k+2 \geq m$, then we have no information coming from the Thom polynomials for higher corank singularities. But Theorem 1.1 gives us $w_{2^{n-1}-m+2} w_{2^{n-1}-m}+w_{2^{n-1}-m+1}^{2}=0$ (with the tangential Stiefel-Whitney classes) if $k+3 \leq 2^{n-1}-m$. This relation does give a restriction because

$w_{2^{n-1}-m+2}\left(\mathbb{R} P^{2^{n}-m}\right) w_{2^{n-1}-m}\left(\mathbb{R} P^{2^{n}-m}\right)+w_{2^{n-1}-m+1}^{2}\left(\mathbb{R} P^{2^{n}-m}\right) \stackrel{(1)}{=} a^{2^{n}-2 m+2}$,

which is nonzero, where $(1)$ comes from the fact that $w_{2^{n-1}-m+1}\left(\mathbb{R} P^{2^{n}-m}\right) \neq 0$ and $w_{2^{n-1}-m+2}\left(\mathbb{R} P^{2^{n}-m}\right)=0$, as we can see by checking the parities of the binomial coefficients $\left(\begin{array}{c}2^{n}-m+1 \\ 2^{n-1}-m+1\end{array}\right)$ and $\left(\begin{array}{c}2^{n}-m+1 \\ 2^{n-1}-m+2\end{array}\right)$; see G199. Thus there exists no Morin map $f$ if $k+3 \leq 2^{n-1}-m$.

As a corollary of Theorem 1.1. we prove

Theorem 1.3. Let $k \geq 0$ and $n=2^{d}+c$ with $0 \leq c<2^{d}-2$ if $c$ is odd.

(1) There is no fold map of $\mathbb{R} P^{n}$ into $S^{n-k}$ for $1 \leq k+1<c$.

(2) There is no Morin map of $\mathbb{R} P^{n}$ into $S^{n-k}$ for $k+2<c$ if $k$ is odd.

For example, there is no Morin map of $\mathbb{R} P^{13}$ into $\mathbb{R}^{12}$, there is no fold map from $\mathbb{R} P^{13}$ to $\mathbb{R}^{j}$ with $10 \leq j \leq 13$, and there is no fold map of $\mathbb{R} P^{11}$ into $\mathbb{R}^{10}$.

Theorem 1.4. If a closed manifold $M^{n}$ admits a corank one map into a stably parallelizable manifold $Q^{n-k}$, then all the rational Pontryagin classes $p_{i}^{\mathbb{Q}}(M) \in$ $H^{4 i}(M ; \mathbb{Q})$ vanish for $i>\frac{1}{2}(k+1)$. 
Remark 1.5. (1) For $n \geq 2$ the class $p_{\lfloor n / 2\rfloor}\left(\mathbb{C} P^{n}\right)$ is equal to $\left(\begin{array}{c}n+1 \\ \lfloor n / 2\rfloor\end{array}\right) y$, where $y$ is the standard generator of $H^{4\lfloor n / 2\rfloor}\left(\mathbb{C} P^{n}\right)$ and hence $p_{\lfloor n / 2\rfloor}^{\mathbb{Q}}\left(\mathbb{C} P^{n}\right)$ does not vanish. Hence there is no corank one map of $\mathbb{C} P^{n}$ to a stably parallelizable target $Q^{2 n-k}$ if $\lfloor n / 2\rfloor \geq(k+2) / 2$. For example, there exist no corank one or Morin maps from $\mathbb{C} P^{2}$ to $Q^{4}$, and from $\mathbb{C} P^{4}$ to $Q^{7}$ (cf. [OSS03, Example 4.9]) or to $Q^{8}$ (cf. [SS98, Theorems 1.2 and 1.3]), and there exists no corank one map of $\mathbb{C} P^{49}$ to $Q^{93}$ (cf. OSS03, Remark 4.5]) if $Q^{i}$ is stably parallelizable.

(2) By Thom transversality and computing the codimension of the Boardman manifolds Boa67, we have that if $n<2(k+2)$, then any manifold $M^{n}$ admits corank one maps into any manifold $Q^{n-k}$. Hence for even $n$, we obtain that $\mathbb{C} P^{n}$ has a corank one map into a stably parallelizable $(2 n-k)$-manifold if and only if $k>n-2$.

1.2. The cobordism class of the source manifold. Combining our results about the relations among characteristic numbers of the source manifold (see Proposition 4.11) with the Dold relations (among characteristic numbers of $n$-manifolds), one gets that the source manifold of a fold map must be unorientedly null-cobordant fairly often or contained in some small-dimensional linear subspaces of the unoriented cobordism group $\mathfrak{N}_{n}$.

For $n \equiv 0 \bmod 4$, let $X^{n}$ be a closed oriented $n$-manifold such that it is unorientedly null-cobordant and its only nonzero Pontryagin characteristic number is $p_{1}^{n / 4}\left[X^{n}\right]>0$, and this is equal to the minimal even value attainable by manifolds with these properties. There exist linear subspaces $\mathfrak{A}^{1}, \mathfrak{B}^{1}$ and $\mathfrak{C}^{2}$ (for their explicit description, see Definition 4.18) of $\mathfrak{N}_{n}$ of dimensions 1, 1 and 2, respectively, such that the following holds:

Theorem 1.6. Let $n \geq 2$. Assume $M$ is a closed oriented $n$-manifold admitting a fold map into a stably parallelizable $(n-1)$-manifold. Then either $M$ is oriented null-cobordant or one of the following cases occurs:

(1) $n \equiv 0 \bmod 4, n$ is not a power of 2 and $M$ is oriented cobordant to $m X^{n}$ for some $m \in \mathbb{Z}$.

(2) $n=2^{a}$ for some $a \geq 2$ and either

(a) $[M]$ is the nonzero element of $\mathfrak{A}^{1}$ or

(b) $M$ is oriented cobordant to $m X^{n}$ for some $m \in \mathbb{Z}$.

(3) $n=2^{b+1}+2^{b}-1$ for some positive integer $b$ and $[M] \in \mathfrak{B}^{1}$.

(4) $n=2^{a}+2^{b}-1$ for some positive integers $a$ and $b, a \geq b+2$, and $[M] \in \mathfrak{C}^{2}$.

Note that in the cases (1) and (2)(b) $M$ is unoriented null-cobordant. Also, the case (2)(a) implies $w_{n}[M] \neq 0$ and can be excluded if $n \neq 2,4,8$; see [SS98] and use the fact that a stably parallelizable manifold is almost parallelizable.

Corollary 1.7. If $n$ is not of the form $2^{a}+2^{b}-1$ for some integers $a>b \geq 0$ and the orientable $n$-manifold $M$ has an odd Pontryagin number or a nonzero StiefelWhitney number, then $M$ has no fold map into any stably parallelizable $(n-1)$ manifold.

Theorem 1.8. Let $n \geq 2$. There exists a 1-dimensional linear subspace $\mathfrak{D}^{1} \leq \mathfrak{N}_{n}$ such that if $M$ is a possibly nonorientable closed n-manifold admitting a tame corank one map into a stably parallelizable $(n-1)$-manifold, then $[M] \in \mathfrak{D}^{1}$ and $w_{1}^{n}[M]=1$ if $M$ is not null-cobordant. 
Proposition 1.9. Let $n>k \geq 5$, where $k$ is odd and not of the form $2^{a}-1$ for some $a \geq 3$. There exists a 1-dimensional linear subspace $\mathfrak{E}^{1} \leq \mathfrak{N}_{n}$ such that if $M$ is a closed $n$-manifold with $w_{1}(M)=\cdots=w_{k}(M)=0$ admitting a fold map into a stably parallelizable $(n-k)$-manifold, then $[M] \in \mathfrak{E}^{1}$. Additionally, if $[M] \in \mathfrak{E}^{1}$ and $M$ is not null-cobordant, then $w_{n}[M]=1$ is the only nonzero monomial characteristic number of $M$.

Again, SS98 implies that $M$ is null-cobordant if $n-k \neq 1,3,7$.

Proposition 1.10. Let $n>k \geq 1$ and $k$ be odd. There exists a 1-dimensional linear subspace $\mathfrak{F}^{1} \leq \mathfrak{N}_{n}$ such that if $M$ is a closed n-manifold which admits a Morin map into a stably parallelizable $(n-k)$-manifold and $w_{i}(M)=0$ for $i=1, \ldots, k+1$, then $[M] \in \mathfrak{F}^{1}$. Additionally, if $[M] \in \mathfrak{F}^{1}$ and $M$ is not null-cobordant, then $w_{n}[M]=1$ is the only nonzero monomial characteristic number of $M$.

As before, the case of $w_{n}[M] \neq 0$ is excluded if $n-k \neq 5,6$ or $n-k \geq 9$; see Sad03, SS98.

Remark 1.11. (1) If $M$ is a spin manifold, then Corollary 1.7 holds with the relaxed condition $n \neq 2,4,8$; see Corollary 4.12 ,

(2) By Theorem 1.6 if an orientable $(4 m+1)$-manifold $M$ admits a fold map into a stably parallelizable $4 m$-manifold, then the de Rham invariant $w_{2} w_{4 m-1}[M]$ vanishes unless $m$ is not a power of 2 .

(3) By Theorem 1.1 we can make statements analogous to the above in the case of not stably parallelizable $Q$ as well.

Numerical calculations similar to those of the proof of Theorem 1.6 suggest the following conjecture:

Conjecture 1.12. Let $n>k \geq 2$ and $k=2^{a}-1$, where $a \geq 2$. There exists a 1-dimensional linear subspace $\mathfrak{G}^{1} \leq \mathfrak{N}_{n}$ such that if a closed $n$-manifold $M$ with $w_{i}(M)=0$ for $i=1, \ldots, k$ admits a fold map into a stably parallelizable $(n-k)$ manifold, then we have one of the following cases:

(1) $n=2^{s}$ or $n=2^{s}+1$ with $s \geq a+1$, and $[M] \in \mathfrak{G}^{1}$.

(2) $M$ is null-cobordant.

We verified this conjecture for $n \leq 1200,3 \leq k \leq 1023$ with the help of a computer.

\subsection{History.}

- It was shown by Ando An04 that there exists a fold map $f: M^{n} \rightarrow Q^{n-k}$ with orientable cokernel bundle $f^{*} T Q / f^{*} d f(T M)$ over the singularity set if and only if there is a bundle epimorphism $T M \oplus \varepsilon^{1} \rightarrow T Q$. Note that if $k$ is even, then the cokernel bundle is automatically orientable.

- Some corollaries of Ando's theorem [An04] were derived in [SSS10.

- More general versions of the results of [An04 are deep theorems stating hprinciples, which are hard to apply directly and led to criteria using Thom polynomials; see for example An85, An87, An01.

- There exist fold maps and cusp maps of $M$ into an almost parallelizable manifold only if the Euler characteristic $\chi(M)$ is even, under the assumption that $n-k$ is large enough [SS98. Refinements of SS98] include results for Morin maps as well when $k$ is odd [An07, Sad03], but nothing is known when $\chi(M)$ is even. 
- For odd $k$, the self-intersection class of the singularity set of a generic corank one map $f$ of $M$ into $Q$ is equal to the $(k+1) / 2$-th Pontryagin class of $T M-f^{*} T Q$ modulo 2-torsion OSS03.

The paper is organized as follows. In $\S 2$ we present and prove the main results about blowing up the source manifold of a singular map. In $\S 3$ we present the main results about K-theoretical characteristic classes. In $\S 4$ we prove the statements of $\S 1$ and $\S 3$.

Conventions. All manifolds henceforth are assumed to be smooth of class $C^{\infty}$. Let $\mathfrak{N}_{n}$ denote the unoriented cobordism group of $n$-dimensional manifolds. The term "cobordant" refers to unoriented cobordism unless oriented cobordism is specified explicitly. For a finite CW-complex $X, \widetilde{K}_{\mathbb{R}}(X)$ and $K_{\mathbb{R}}(X)$ denote the reduced and unreduced real K-rings of $X$, respectively, with $\widetilde{K}_{\mathbb{R}}(X) \subseteq K_{\mathbb{R}}(X)$. The symbol $\varepsilon_{X}^{n}$ denotes the trivial $n$-dimensional bundle over the space $X$, and the lower index " $X$ " will be omitted when it is clear from the context. Wherever not stated otherwise, we use the convention that if $\beta<0$ or $\alpha<\beta$, then the binomial coefficient $\left(\begin{array}{l}\alpha \\ \beta\end{array}\right)=0$.

\section{BLOWING UP THE SOURCE MANIFOLD ALONG THE SINGULARITY SET}

Here we shall consider Morin maps $f: M^{n} \rightarrow Q^{n-k}$, where $n>k \geq 0, M$ is closed, and $Q$ is an arbitrary smooth manifold. It is well known that

(1) All of the singularities of a Morin map are Morin singularities, i.e., singularities of types $\Sigma^{k+1,1, \ldots, 1,0}$ by using Thom-Boardman symbols.

(2) Morin maps are stable corank one maps.

(3) The set of singular points is an embedded $(n-k-1)$-dimensional manifold.

(4) Denoting by $\Sigma^{I}=\Sigma^{I}(f)$ the set of corresponding points for a ThomBoardman symbol $\Sigma^{I}$ one has that the closure of $\Sigma^{k+1,1, \ldots, 1,0}$ is $\Sigma^{k+1,1, \ldots, 1}$ (the same number of digits 1 ). In the chain of embedded submanifolds $\Sigma^{k+1} \supset \Sigma^{k+1,1} \supset \cdots$ the dimensions decrease by one.

The points in $\Sigma^{k+1,0}$ are called fold points, and those in $\Sigma^{k+1,1,0}$ are cusp points. A Morin map is called fold map, respectively cusp map, if it has beside the regular points only fold points, respectively fold and cusp points. We note that in general a corank one map cannot be perturbed to obtain a Morin map.

Definition 2.1 (Blowup). Recall that given an embedded closed submanifold $V$ in $M$, the blowup of $M$ along $V$ is a well-known construction. Denoting the normal bundle of $V$ by $\zeta$, it produces a manifold $\mathrm{Bl}_{\zeta} M$ and a projection $\pi: \mathrm{Bl}_{\zeta} M \rightarrow M$ such that $\pi^{-1}(V)$ is the projectivization of $\zeta$ and outside $\pi^{-1}(V)$ the projection $\pi$ is a diffeomorphism onto $M-V$. If $f: M \rightarrow Q$ is any smooth map, then we denote by $\mathrm{Bl}_{\zeta} f$ the composition $f \circ \pi$.

Remark 2.2. Let $f: M^{n} \rightarrow Q^{n-k}$ be a generic corank one map. The singularity set $\Sigma$ is an embedded $(n-k-1)$-dimensional submanifold of $M$ [Boa67]; denote its normal bundle by $\xi$. Then the map $\mathrm{Bl}_{\xi} f$ is a nongeneric corank one map and its singularity set is $\pi^{-1}(\Sigma)$.

Lemma 2.3. For any submanifold $V$ in the closed manifold $M$ the projection $\pi$ induces monomorphisms $\pi^{*}: H^{m}\left(M ; \mathbb{Z}_{2}\right) \rightarrow H^{m}\left(\mathrm{Bl}_{\zeta} M ; \mathbb{Z}_{2}\right)$ of the cohomology groups with $\mathbb{Z}_{2}$ coefficients. 
Proof. Consider the Gysin map $\pi_{!}: H^{m}\left(\mathrm{Bl}_{\zeta} M ; \mathbb{Z}_{2}\right) \rightarrow H^{m}\left(M ; \mathbb{Z}_{2}\right)$. Denote by PD the Poincaré duality map PD: $H_{n}\left(M^{n} ; \mathbb{Z}_{2}\right) \rightarrow H^{0}\left(M^{n} ; \mathbb{Z}_{2}\right)$. Then for any $x \in H^{m}\left(M ; \mathbb{Z}_{2}\right)$ we have

$$
\begin{aligned}
\pi_{!}\left(\pi^{*}(x)\right) & =\pi_{!}\left(\pi^{*}(x) \cup 1\right)=x \cup \pi_{!}(1)=x \cup \operatorname{PD}\left(\pi_{*}\left(\left[\mathrm{Bl}_{\zeta} M\right] \cap 1\right)\right) \\
& =x \cup \operatorname{PD}\left(\pi_{*}\left(\left[\mathrm{Bl}_{\zeta} M\right]\right)\right)=x \cup \operatorname{PD}([M])=x \cup 1=x .
\end{aligned}
$$

Let $f: M^{n} \rightarrow Q^{n-k}$ be a Morin map. It is well known that in a neighborhood of each point $p$ of $\Sigma^{k+1,0}$ the map $f$ can be written in the form

$$
\left(x_{1}, \ldots, x_{n}\right) \mapsto\left(\sum_{i=1}^{k+1-\lambda} x_{i}^{2}-\sum_{i=k+2-\lambda}^{k+1} x_{i}^{2}, x_{k+2}, \ldots, x_{n}\right),
$$

where $0 \leq \lambda \leq(k+1) / 2$ and $\lambda$ is called the index of the fold singularity $p$.

Let $\Sigma_{\lambda}^{\bar{k}+1,0}$ denote the set of fold singular points of index $\lambda$ of $f$. We denote the $(k+1)$-dimensional normal bundle of the singularity set $\Sigma=\Sigma^{k+1}$ of $f$ by $\xi$. Let $k$ be odd and denote by $\eta$ the restriction of $\xi$ to $\Sigma_{(k+1) / 2}^{k+1,0}$. Then, the normal bundle $\eta$ has structure group $G(\eta)$ generated by transformations of the form

$$
\left(x_{1}, \ldots, x_{k+1}\right) \mapsto A\left(x_{1}, \ldots, x_{k+1}\right) \text { with } A \in O\left(\frac{k+1}{2}\right) \times O\left(\frac{k+1}{2}\right)
$$

and

$$
\left(x_{1}, \ldots, x_{k+1}\right) \mapsto\left(x_{(k+1) / 2+1}, \ldots, x_{k+1}, x_{1}, \ldots, x_{(k+1) / 2}\right) .
$$

The restriction of $f$ to any fiber of $\eta$ is mapped into a single fiber of the normal line bundle of $f(\Sigma)$ in $Q$ and it is left-right equivalent to the saddle singularity

$$
\left(x_{1}, \ldots, x_{k+1}\right) \mapsto y=\sum_{i=1}^{(k+1) / 2} x_{i}^{2}-\sum_{i=(k+1) / 2+1}^{k+1} x_{i}^{2},
$$

i.e., to a Morse function of index $(k+1) / 2$.

We call a corank one map $f: M \rightarrow Q$ tame if the 1-dimensional cokernel bundle coker $\left.d f\right|_{\Sigma}$ of the restriction $\left.d f\right|_{\Sigma}:\left.T M\right|_{\Sigma} \rightarrow f^{*} T Q$ is trivial. For example, every fold map is tame for $k \equiv 0 \bmod 2$ [An04 and it is easy to construct not tame fold maps for odd $k \leq n-3$, even between orientable manifolds.

We recall the notion of the Morse-Bott map. Roughly, this is a smooth map which is locally the product $p \times h: \mathbb{R}^{q} \times \mathbb{R}^{q^{\prime \prime}} \rightarrow \mathbb{R}^{q^{\prime}} \times \mathbb{R}$ of a submersion $p: \mathbb{R}^{q} \rightarrow \mathbb{R}^{q^{\prime}}$ and a Morse function $h: \mathbb{R}^{q^{\prime \prime}} \rightarrow \mathbb{R}$, but the values $q$ and $q^{\prime \prime}$ might be different for different singularity points (lying in different components of the singularity set).

Definition 2.4 (Morse-Bott map). For $n>k \geq 0$, a map $g: P^{n} \rightarrow Q^{n-k}$ of a manifold $P$ is a Morse-Bott map if

(1) the set of singular points $\Sigma$ of $g$ is the disjoint union $\bigsqcup_{i} S_{i}$ of smooth closed connected submanifolds $S_{i}$ of $P$,

(2) each component $S_{i}$ is the total space of a smooth bundle whose fiber is a connected manifold $C_{i}$,

(3) further, for each component $S_{i}$ there exist

(a) numbers $\lambda$ and $l$ such that $0 \leq \lambda \leq l \leq k+1$ and

\footnotetext{
${ }^{2}$ Note that the index of a fold singularity is well-defined.
} 
(b) for each $p \in S_{i}$ there are neighborhoods $U_{1}$ of $p$ in $P$ and $U_{2}$ of $g(p)$ in $Q$, and there are diffeomorphisms

$$
u_{1}: U_{1} \rightarrow \mathbb{R}^{l} \times \mathbb{R}^{k+1-l} \times \mathbb{R}^{n-k-1} u_{2}: U_{2} \rightarrow \mathbb{R} \times \mathbb{R}^{n-k-1}
$$

such that

(i) $u_{1}(p)=0, u_{2}(g(p))=0$,

(ii) $u_{1}\left(U_{1} \cap \Sigma\right)=\left\{(x, y, z) \in \mathbb{R}^{l} \times \mathbb{R}^{k+1-l} \times \mathbb{R}^{n-k-1}: x=0\right\}$,

(iii) for the fiber $C_{i}$ containing $p, u_{1}\left(U_{1} \cap C_{i}\right)=\left\{(x, y, z) \in \mathbb{R}^{l} \times\right.$ $\left.\mathbb{R}^{k+1-l} \times \mathbb{R}^{n-k-1}: x=0, z=0\right\}$, and

(iv) $u_{2} \circ g \circ u_{1}^{-1}(x, y, z)=\left(\sum_{i=1}^{\lambda}-x_{i}^{2}+\sum_{i=\lambda+1}^{l} x_{i}^{2}, z\right)$.

The index of a Morse-Bott map $g$ at a singular point $p$ is the pair $(\lambda, k+1-l)$ if $\lambda \leq l-\lambda$.

Remark 2.5. Compare Definition 2.4 with [BH04, the Morse-Bott Lemma].

Note that for a Morse-Bott map $g: P \rightarrow Q$ the index at a singular point is well-defined. Let $\Sigma_{(\lambda, k+1-l)}=\Sigma_{(\lambda, k+1-l)}(g)$ denote the set of singular points of $g$ which have index $(\lambda, k+1-l)$. Then $\Sigma_{(\lambda, k+1-l)}$ is an $(n-l)$-dimensional closed submanifold of $P$. Note that a Morse-Bott map is a corank one map, although it is not necessarily generic or Morin.

For each index $(\lambda, k+1-l)$, let $\tilde{\Sigma}_{(\lambda, k+1-l)}=\tilde{\Sigma}_{(\lambda, k+1-l)}(g)$ denote the quotient manifold $\Sigma_{(\lambda, k+1-l)} / \sim$, where $p \sim q$ if and only if $p$ and $q$ lie in the same connected fiber $C_{i}$ for some $i$. Clearly $\tilde{\Sigma}_{(\lambda, k+1-l)}$ is an $(n-k-1)$-dimensional manifold. The continuous map $\tilde{g}_{(\lambda, k+1-l)}: \tilde{\Sigma}_{(\lambda, k+1-l)} \rightarrow Q$ determined by the property $g=\tilde{g}_{(\lambda, k+1-l)} \circ q_{g}$, where $q_{g}: \Sigma_{(\lambda, k+1-l)} \rightarrow \tilde{\Sigma}_{(\lambda, k+1-l)}$ is the quotient map, is an immersion. Put $\tilde{\Sigma}=\bigcup \tilde{\Sigma}_{(\lambda, k+1-l)}$ and $\tilde{g}=\bigcup \tilde{g}_{(\lambda, k+1-l)}$, i.e. $\left.\tilde{g}\right|_{\tilde{\Sigma}_{(\lambda, k+1-l)}}=\tilde{g}_{(\lambda, k+1-l)}$. Note that $q_{g}$ and $\tilde{g}$ give just the Stein factorization of $\left.g\right|_{\Sigma}$.

The cokernel bundle $\left.\left(g^{*} T Q / g^{*} d f(T P)\right)\right|_{\Sigma}$ can be identified with the pull-back $q_{g}^{*} \tilde{g}^{*} \nu$, where $\nu$ is the normal bundle of the immersion $\tilde{g}: \tilde{\Sigma} \rightarrow Q$.

If $\lambda \neq l-\lambda$, then the 1-dimensional normal bundle of the immersion $\tilde{g}_{(\lambda, k+1-l)}$ is clearly trivial. Also note that a Morse-Bott map $g$ is tame if and only if all the normal bundles of the immersions $\tilde{g}_{(\lambda, k+1-l)}$ are trivial.

Recall that for a fold map $f: M^{n} \rightarrow Q^{n-k}$ with odd $k$, we denote by $\eta$ the $(k+1)$-dimensional normal bundle of $\Sigma_{(k+1) / 2}^{k+1,0}$.

Theorem 2.6. Let $k \geq 1$ be odd and $f: M^{n} \rightarrow Q^{n-k}$ be a fold map. The map

$$
\mathrm{Bl}_{\eta} f: \mathrm{Bl}_{\eta} M \rightarrow Q
$$

can be perturbed in a neighborhood of $\pi^{-1}\left(\Sigma_{(k+1) / 2}^{k+1,0}\right)$ to obtain a $\Theta: \mathrm{Bl}_{\eta} M \rightarrow Q$ tame Morse-Bott map. The stable tangent bundle of $\mathrm{Bl}_{\eta} M$ splits as

$$
T \mathrm{Bl}_{\eta} M \oplus \varepsilon^{1} \cong \zeta^{k+1} \oplus \Theta^{*} T Q
$$

for some $(k+1)$-dimensional vector bundle.

This theorem also has an analogue in the case when $f: M \rightarrow Q$ is any Morin map. Recall that we denote the $(k+1)$-dimensional normal bundle of the singularity set $\Sigma(f)$ by $\xi$.

Theorem 2.7. Let $k \geq 1$ be odd and $f: M^{n} \rightarrow Q^{n-k}$ be a Morin map. The map

$$
\mathrm{Bl}_{\xi} f: \mathrm{Bl}_{\xi} M \rightarrow Q
$$


can be perturbed in a neighborhood of $\pi^{-1}\left(\Sigma_{(k+1) / 2}^{k+1,0}\right)$ to obtain a map $\Theta: \mathrm{Bl}_{\xi} M \rightarrow$ $Q$ such that the stable tangent bundle of $\mathrm{Bl}_{\xi} M$ splits as

$$
T \mathrm{Bl}_{\xi} M \oplus \varepsilon^{2} \cong \zeta^{k+2} \oplus \Theta^{*} T Q
$$

for some $(k+2)$-dimensional vector bundle.

Proof of Theorem 2.6. Let $\nu$ denote the 1-dimensional normal bundle of the immersion

$$
\left.f\right|_{\Sigma_{(k+1) / 2}^{k+1,0}}: \Sigma_{(k+1) / 2}^{k+1,0} \rightarrow Q .
$$

We identify the normal bundle $\eta$ with a tubular neighborhood of $\Sigma_{(k+1) / 2}^{k+1,0}$ in $M$ in such a way that $f$ restricted to $\eta$ is a composition of

(1) a (nonlinear) bundle map $\iota: \eta \rightarrow \nu$ having the form

$$
\left(x_{1}, \ldots, x_{k+1}\right) \mapsto y=\sum_{i=1}^{(k+1) / 2} x_{i}^{2}-\sum_{i=(k+1) / 2+1}^{k+1} x_{i}^{2}
$$

on the unit disk of each fiber of $\eta$ in suitable local coordinates and (2) an immersion $\varphi: \nu \rightarrow Q$.

Under this identification, the points of $\mathrm{Bl}_{\eta} M$ in the neighborhood $\pi^{-1}(\eta)$ are identified with the sets of pairs $[(v, \tilde{v})]:=\{(v, \tilde{v}),(v,-\tilde{v})\}$, where $v$ and $\tilde{v}$ are parallel vectors in the same fiber of $\eta$ and $\tilde{v}$ has length 1 . Further $\mathrm{Bl}_{\eta} f([(v, \tilde{v})])$ coincides with $\varphi \circ \iota(v)$ under these identifications.

We define the perturbation $\Theta$ of $\mathrm{Bl}_{\eta} f$ as follows:

(1) It coincides with $\mathrm{Bl}_{\eta} f$ outside $\pi^{-1}(\eta) \subset \mathrm{Bl}_{\eta} M$.

(2) If $p \in \Sigma_{(k+1) / 2}^{k+1,0}, v, \tilde{v} \in \eta_{p}$ are collinear vectors, and $\|\tilde{v}\|=1$, then $p^{\prime}=[(v, \tilde{v})]$ is a point in $\pi^{-1}(\eta)$. Let $\varepsilon: \Sigma_{(k+1) / 2}^{k+1,0} \rightarrow[0, \infty)$ be a small smooth function which will be defined later and let $\omega:[0,1] \rightarrow[0,1]$ be a smooth "bump function": $\omega(\cdot)=1, \omega(\cdot)=0$ on neighborhoods of 0 and 1 , respectively. Now put

$$
\Theta\left(p^{\prime}\right)=\varphi(\iota(v)+\varepsilon(p) \omega(\|v\|) \iota(\tilde{v})) .
$$

Note that replacing $\tilde{v}$ by $-\tilde{v}$ on the right hand side does not change since $\iota(\tilde{v})=\iota(-\tilde{v})$ (this is necessary for the correctness of the definition since $p^{\prime}$ could be represented by $([v,-\tilde{v}])$ as well).

Lemma 2.8. Let $p \in \Sigma_{(k+1) / 2}^{k+1,0}$. Then the singular points of the function

$$
\left.\Theta\right|_{\pi^{-1}\left(\eta_{p}\right)}: \pi^{-1}\left(\eta_{p}\right) \rightarrow \nu_{f(p)}
$$

are contained in $\pi^{-1}(p)$.

Proof. Let $\tilde{v} \in \eta_{p},\|\tilde{v}\|=1$. First let us suppose that $\iota(\tilde{v}) \neq 0$. Take a curve $\gamma(t)=[(t \tilde{v}, \tilde{v})]$ in the blowup $\pi^{-1}\left(\eta_{p}\right)$. Then the composition

$$
\Theta \circ \gamma(t)=\varphi(\iota(t \tilde{v})+\varepsilon(p) \omega(t) \iota(\tilde{v}))=\varphi\left(\left(t^{2}+\varepsilon(p) \omega(t)\right) \iota(\tilde{v})\right)
$$

has a critical point only at $t=0$ if $\varepsilon(p)$ is small enough. Hence a point $[(t \tilde{v}, \tilde{v})] \in$ $\pi^{-1}\left(\eta_{p}\right)$ cannot be a critical point of the function $\left.\Theta\right|_{\pi^{-1}\left(\eta_{p}\right)}: \pi^{-1}\left(\eta_{p}\right) \rightarrow \nu_{f(p)}$ if $t \neq 0, \iota(\tilde{v}) \neq 0$ and $\varepsilon(p)$ is small enough. 

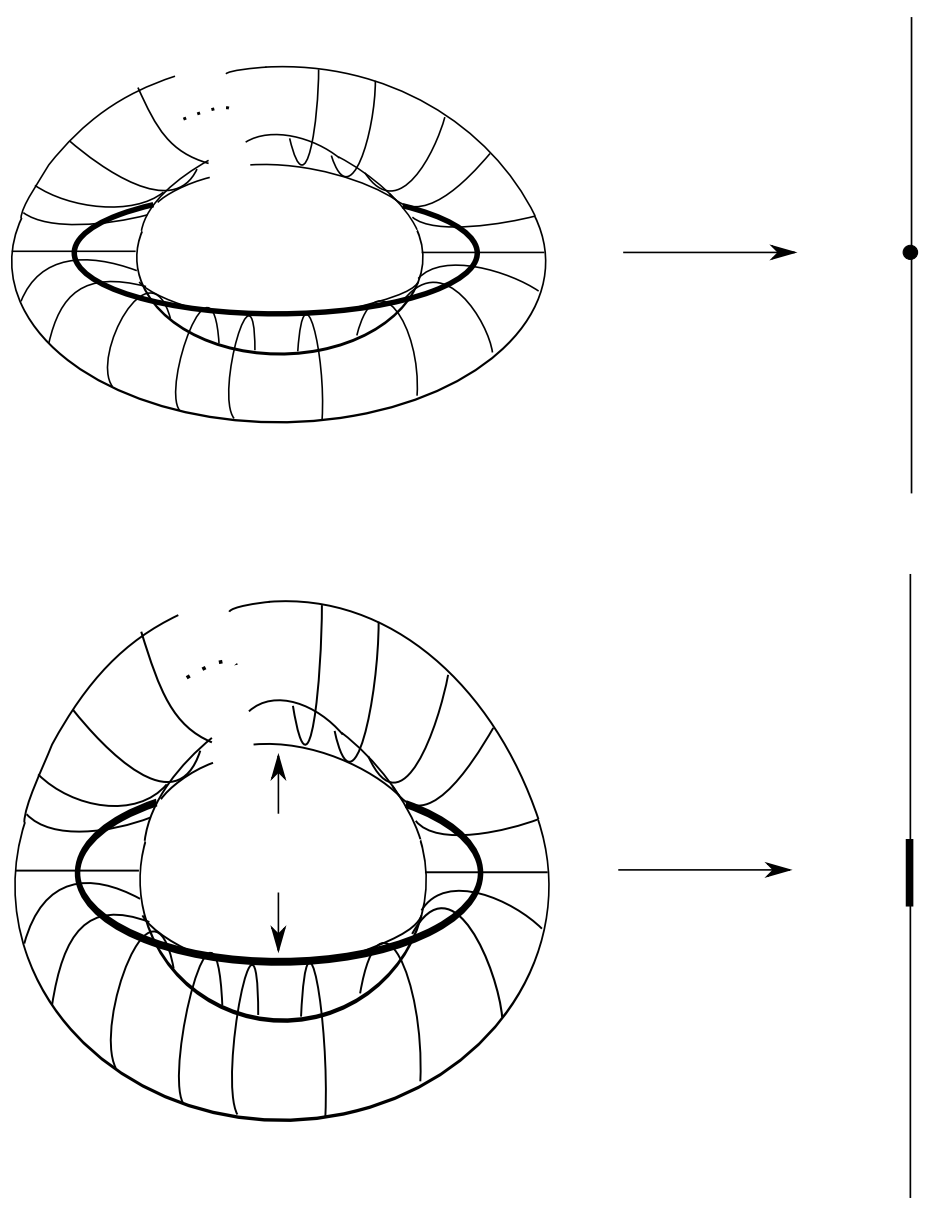

FiguRE 1. The function $\left.\mathrm{Bl}_{\eta} f\right|_{\pi^{-1}\left(\eta_{p}\right)}: \pi^{-1}\left(\eta_{p}\right) \rightarrow \nu_{f(p)}$ and its perturbation in the case of $k=1$. Note that $\pi^{-1}\left(\eta_{p}\right)$ is a Möbius band. In the upper picture the bold circle (i.e. the centerline of the Möbius band) is mapped into one point, while in the lower picture it is mapped into a small interval because of the perturbation.

Now assume that $\iota(\tilde{v})=0$. For a fixed $t_{0}$, take a curve $\delta(s)=\left[\left(t_{0} \tilde{v}_{s}, \tilde{v}_{s}\right)\right]$ such that $\tilde{v}_{0}=\tilde{v}$ and $s \mapsto \delta(s)$ intersects $\{[(v, \tilde{v})]: \iota(\tilde{v})=0\}$ transversally. Then the composition

$$
\Theta \circ \delta(s)=\varphi\left(\iota\left(t_{0} \tilde{v}_{s}\right)+\varepsilon \omega\left(t_{0}\right) \iota\left(\tilde{v}_{s}\right)\right)=\varphi\left(\left(t_{0}^{2}+\varepsilon \omega\left(t_{0}\right)\right) \iota\left(\tilde{v}_{s}\right)\right)
$$

has nonzero derivative at $s=0$. Hence the function $\left.\Theta\right|_{\pi^{-1}\left(\eta_{p}\right)}: \pi^{-1}\left(\eta_{p}\right) \rightarrow \nu_{f(p)}$ is regular at a point $[(t \tilde{v}, \tilde{v})] \in \pi^{-1}\left(\eta_{p}\right)$ if $\iota(\tilde{v})=0$. This completes the proof of Lemma 2.8

Lemma 2.9. The singularity sets of the restrictions of $\Theta$ to $\pi^{-1}(p)$ and $\pi^{-1}\left(\eta_{p}\right)$ are equal $\left(\Theta\right.$ is considered in both cases as a function to $\left.\nu_{f(p)}\right)$. 
Proof. Let us denote by $\Sigma(h)$ the singularity set of a map $h$. For negative codimension, singularity means that the differential is not epimorphic in the given point. Hence by Lemma 2.8 the inclusion $\Sigma\left(\left.\Theta\right|_{\pi^{-1}(p)}\right) \supset \Sigma\left(\left.\Theta\right|_{\pi^{-1}\left(\eta_{p}\right)}\right)$ is obvious since $\pi^{-1}(p) \subset \pi^{-1}\left(\eta_{p}\right)$.

Next, we show the opposite, less trivial inclusion. The tangent vectors of lines $\gamma(t)=[(t \tilde{v}, \tilde{v})]$ at $t=0$ generate the normal fibers of the embedding $\pi^{-1}(p) \subset$ $\pi^{-1}\left(\eta_{p}\right)$. So it is enough to show that for any such line the composition $\Theta \circ \gamma(t)$ has vanishing differential at $t=0$ if the corresponding point is critical for the function $\left.\Theta\right|_{\pi^{-1}(p)}: \pi^{-1}(p) \rightarrow \nu_{f(p)}$.

The choice of local coordinates $x_{1}, \ldots, x_{k+1}$ on $\eta_{p}$ identifies $\pi^{-1}(p)$ with the projective space $\mathbb{R} P^{k}$, and the restriction $\left.\Theta\right|_{\pi^{-1}(p)}$ is equal to

$$
\left.\Theta\right|_{\pi^{-1}(p)}:\left[x_{1}: \cdots: x_{k+1}\right] \mapsto \varphi\left(\varepsilon(p) \iota\left(\frac{\left(x_{1}, \ldots, x_{k+1}\right)}{\left\|\left(x_{1}, \ldots, x_{k+1}\right)\right\|}\right)\right) .
$$

This map is Morse-Bott and has critical points along two copies of $\mathbb{R} P^{(k+1) / 2-1}$, which are $\left\{\left[x_{1}: \cdots: x_{(k+1) / 2}: 0: \cdots: 0\right] \in \mathbb{R} P^{k}\right\}$ and $\left\{\left[0: \cdots: 0: x_{(k+1) / 2+1}: \cdots:\right.\right.$ $\left.\left.x_{k+1}\right] \in \mathbb{R} P^{k}\right\}$. Along these two copies of $\mathbb{R} P^{(k+1) / 2-1}$, since the function $\iota$ does not take the value 0 , by the proof of the previous lemma we have that $\Theta \circ \gamma(t)$ has vanishing differential.

It is easy to see that both critical loci of $\left.\Theta\right|_{\pi^{-1}(p)}$ have index $(0,(k+1) / 2-1)$. Therefore by the above lemmas $\left.\Theta\right|_{\pi^{-1}\left(\eta_{p}\right)}$ is a Morse-Bott map with indices $(1,(k+$ $1) / 2-1)$ and with these two copies of $\mathbb{R} P^{(k+1) / 2-1}$ as the singularity set.

The singularity set $\Sigma\left(\left.\Theta\right|_{\pi^{-1}(\eta)}\right)$ is the union for each $p \in \Sigma_{(k+1) / 2}^{k+1,0}$ of the singularity set of the restriction of $\Theta$ to the fiber $\eta_{p}$, i.e.,

$$
\Sigma\left(\left.\Theta\right|_{\pi^{-1}(\eta)}\right)=\bigcup_{p \in \Sigma_{(k+1) / 2}^{k+1,0}} \Sigma\left(\left.\Theta\right|_{\pi^{-1}\left(\eta_{p}\right)}\right) .
$$

Moreover, mapping each set $\Sigma\left(\left.\Theta\right|_{\pi^{-1}\left(\eta_{p}\right)}\right)$ to the point $p \in \Sigma_{(k+1) / 2}^{k+1,0}$ we obtain a fiber bundle $\Sigma\left(\left.\Theta\right|_{\pi^{-1}(\eta)}\right) \rightarrow \Sigma_{(k+1) / 2}^{k+1,0}$ with fiber $\Sigma\left(\left.\Theta\right|_{\pi^{-1}\left(\eta_{p}\right)}\right)$. Hence $\Theta$ is a Morse-Bott map with the corresponding indices.

Recall that the singularity set $\Sigma(\Theta)$ of $\Theta$ consists of two disjoint projective spaces in each fiber of the projective bundle $P(\eta) \rightarrow \Sigma_{(k+1) / 2}^{k+1,0}$ associated to the vector bundle $\eta$. When we form the Stein factorization ${ }^{3}$ of the restriction $\left.\Theta\right|_{\Sigma(\Theta)}$, we pinch each of these projective spaces into one point. Hence we obtain a double cover over $\Sigma_{(k+1) / 2}^{k+1,0}$. This double cover is precisely the one associated to the normal line bundle $\nu$ of $\left.f\right|_{\Sigma_{(k+1) / 2}^{k+1,0}}$. The map $\Theta$ defines an immersion of this Stein factorization into $Q$ whose normal bundle $\tilde{\nu}$ is trivial since it is the lift of $\nu$ to the double cover associated to it. Moreover, there is a natural trivialization of $\tilde{\nu}$ corresponding to the indices of the singularity set of $\left.\Theta\right|_{\pi^{-1}(p)}, p \in \Sigma_{(k+1) / 2}^{k+1,0}$.

Thus the perturbation $\Theta$ satisfies the requirements of the theorem. Hence $\Theta$ is a tame corank one map, and applying the trivial direction $(1) \Longrightarrow(2)$ of Proposition 3.1 finishes the proof.

Remark 2.10. The constructed double covering of $\Sigma_{(k+1) / 2}^{k+1,0}$ is trivial if and only if the bundle $\nu$ is trivial.

\footnotetext{
${ }^{3}$ See Remark 2.5
} 
Remark 2.11. If $k=1$, then $\Theta$ is obviously a fold map. For example, for a Morse function $f: S^{2} \rightarrow \mathbb{R}$ with three definite and one indefinite critical points, $\mathrm{Bl}_{\eta} S^{2}=$ $S^{2} \# \mathbb{R} P^{2}=\mathbb{R} P^{2}$, and $\Theta$ is a Morse function with three definite and two indefinite critical points. It can be seen that $\Theta$ has two singular fibers containing indefinite critical points and that exactly one of them has a nonorientable neighborhood.

Lemma 2.12. Let $X$ be a manifold and $l$ be a line bundle over $X$. Assume that there is an open covering $X_{0} \cup X_{1}=X$ such that the bundle $l$ is trivial over both $X_{0}$ and $X_{1}$. Then there exists an epimorphism $\varepsilon_{X}^{2} \rightarrow l$.

Proof. Details are left to the reader.

Proof of Theorem [2.7. Suppose $f: M^{n} \rightarrow Q^{n-k}$ is a Morin map. By [Ch83] the cokernel bundle of $d f: T M \rightarrow f^{*} T Q$ is trivial over a neighborhood of $\Sigma^{k+1,1}$ in $\Sigma$. Moreover, coker $d f$ has natural orientations over the fold singularities of index not equal to $(k+1) / 2$, and these orientations agree when two fold singularity sets are attached to each other along $\Sigma^{k+1,1}$. Let $\Sigma_{\geq 0}$ denote the complement of a small regular neighborhood of $\Sigma^{k+1,1} \cup \bigcup_{\lambda \neq(k+1) / 2} \Sigma_{\lambda}^{k+1,0}$ in $\Sigma$. Then coker $d f$ is trivial on $\Sigma-\Sigma_{\geq 0}$. If $\Sigma_{\geq 0}=\emptyset$, then $f$ is a tame corank one map, and by Proposition 3.1 we obtain the statement of the theorem. Assume that $\Sigma_{\geq 0} \neq \emptyset$.

Take the blowup $\mathrm{Bl}_{\xi} f: \mathrm{Bl}_{\xi} M \rightarrow Q$. Define a smooth nonnegative function $\Delta$ on $\Sigma_{\geq 0}$ such that it vanishes on $\Sigma_{=0}$, where $\Sigma_{=0}$ is a small compact regular neighborhood of $\partial \Sigma_{\geq 0}$, and takes small positive values on $\Sigma_{>0}$, where $\Sigma_{>0}=\Sigma_{\geq 0}-$ $\Sigma_{=0}$. Then, perturb the map $\mathrm{Bl}_{\xi} f: \mathrm{Bl}_{\xi} M \rightarrow Q$ in $\pi^{-1}\left(\left.\eta\right|_{\Sigma_{\geq 0}}\right)$ in the same way as we did in Theorem 2.6. but replace $\varepsilon$ in (2.3) by the value of the function $\Delta$. Denote the resulting map by $\widetilde{\mathrm{Bl}_{\xi} f}$. Note that on $\mathrm{Bl}_{\xi} M-\pi^{-1}\left(\left.\eta\right|_{\Sigma-\Sigma_{>0}}\right)$ the restrictions of $\widetilde{\mathrm{Bl}_{\xi} f}$ and $\mathrm{Bl}_{\xi} f$ coincide. Denote by $\tilde{\Sigma}_{>0}$ the singularity set of $\left.\widetilde{\mathrm{Bl}_{\xi} f}\right|_{\pi^{-1}\left(\left.\eta\right|_{\Sigma_{>0}}\right)}$. The singularity set of $\left.\left.\widetilde{\mathrm{Bl}_{\xi} f}\right|_{\pi^{-1}\left(\left.\xi\right|_{\Sigma-\Sigma}>0\right.}\right)$ is equal to $\pi^{-1}\left(\Sigma-\Sigma_{>0}\right)$.

Since $d \mathrm{Bl}_{\xi} f\left(T \mathrm{Bl}_{\xi} M\right)=d f d \pi\left(T \mathrm{Bl}_{\xi} M\right)=d f(T M)$ and $f^{*} T Q / f^{*} d f(T M)$ is trivial on $\Sigma-\Sigma_{>0}$, clearly coker $d \mathrm{Bl}_{\xi} f=\pi^{*} f^{*}(T Q) / \pi^{*} f^{*} d \mathrm{Bl}_{\xi} f\left(T \mathrm{Bl}_{\xi} M\right)$ is also trivial on $\pi^{-1}\left(\Sigma-\Sigma_{>0}\right)$. Hence so is coker $\widetilde{\mathrm{Bl}_{\xi} f}$. Moreover, $d \widetilde{\mathrm{Bl}_{\xi} f}$ also has trivial cokernel on $\tilde{\Sigma}_{>0}$ by Theorem 2.6.

Apply Lemma 2.12 to $l=$ coker $d \widetilde{\mathrm{Bl}_{\xi} f}$ over

$$
X=\tilde{\Sigma}_{>0} \cup \pi^{-1}\left(\Sigma-\Sigma_{>0}\right)
$$

with the covering consisting of $X_{0}=\tilde{\Sigma}_{>0}$ and $X_{1}$ being a small neighborhood of $\pi^{-1}\left(\Sigma-\Sigma_{>0}\right)$. The argument above ensures that $l$ is indeed trivial when restricted to either $X_{0}$ or $X_{1}$ since $X_{1}$ is a deformation retract of $\pi^{-1}\left(\Sigma-\Sigma_{>0}\right)$. Therefore there exists a fiberwise epimorphism $\sigma:\left.\varepsilon_{X}^{2} \rightarrow \operatorname{coker} d \widetilde{\mathrm{Bl}_{\xi} f}\right|_{X}$. Compose $\sigma$ with the standard embedding coker $\left.\left.d \widetilde{\mathrm{Bl}_{\xi} f}\right|_{X} \rightarrow\left(\widetilde{\mathrm{Bl}_{\xi} f}\right)^{*} T Q\right|_{X}$ and then extend this composite map to all of $\mathrm{Bl}_{\xi} M$ as a linear bundle map $\tilde{\sigma}: \varepsilon_{\mathrm{Bl}_{\xi} M}^{2} \rightarrow\left(\widetilde{\mathrm{Bl}_{\xi} f}\right)^{*} T Q$ by scaling it with a bump function concentrated on a small neighborhood of $X$. Combining $\widetilde{\mathrm{Bl}_{\xi} f}$ with $\tilde{\sigma}$ we get a bundle map

$$
d \widetilde{\mathrm{Bl}_{\xi} f}+\tilde{\sigma}: T \mathrm{Bl}_{\xi} M \oplus \varepsilon^{2} \rightarrow\left(\widetilde{\mathrm{Bl}_{\xi} f}\right)^{*} T Q,
$$

which is obviously surjective both on $\mathrm{Bl}_{\xi} M-X$ and $X$. This completes the proof. 


\section{K-THEORETICAL CHARACTERISTIC CLASSES}

From the viewpoint of K-theory and $\gamma$ operations, we have the following 4 Recall that for a finite $\mathrm{CW}$-complex $X$ the geometric dimension $g \cdot \operatorname{dim}(x)$ of an element $x \in \widetilde{K}_{\mathbb{R}}(X)$ is the least integer $k$ such that $x+k$ is a class of a genuine vector bundle over $X$ (see e.g. At61]).

Let $M^{n}$ and $Q^{n-k}$ be a closed $n$-manifold and an $(n-k)$-manifold, respectively.

Proposition 3.1. The following are equivalent:

(1) $M$ admits a tame corank one map into $Q$,

(2) there is a fiberwise epimorphism $T M \oplus \varepsilon^{1} \rightarrow T Q$.

If $Q$ is stably parallelizable, then (1) and (2) hold if and only if g.dim $\left([T M]-\left[\varepsilon^{n}\right]\right) \leq$ $k+1$.

Let $\lambda_{t}=\sum_{i=0}^{\infty} \lambda^{i} t^{i}$, where $\lambda^{i}$ are the exterior power operators (for details, see At61]). For a finite CW-complex $X$, define $\gamma_{t}=\sum_{i=0}^{\infty} \gamma^{i} t^{i}$ to be the homomorphism $\lambda_{t / 1-t}$ of $K_{\mathbb{R}}(X)$ into the multiplicative group of formal power series in $t$ with coefficients in $K_{\mathbb{R}}(X)$ and constant term 1. By the above proposition and At61, Proposition 2.3], we immediately have

Corollary 3.2.5 If $M^{n}$ admits a tame corank one map into a stably parallelizable $Q^{n-k}$, then

(1) $w_{i}(M)=0$ for $i \geq k+2$,

(2) $p_{i}(M)=0$ for $2 i>k+1$,

(3) $\gamma^{i}\left([T M]-\left[\varepsilon^{n}\right]\right)=0$ for $i \geq k+2$.

Remark 3.3. Note that conditions (1) and (2) may not give strong results in general: for example, all the positive degree Stiefel-Whitney and Pontryagin classes of $\mathbb{R} P^{2^{n}-1} \operatorname{vanish} 6$ and if $k+1 \geq n / 2$, then condition (2) is satisfied trivially for any $M$. In particular cases, though, condition (1) can still give strong results, e.g. all the Stiefel-Whitney classes of $\mathbb{R} P^{2^{n}-2}$ of degree up to $2^{n}-2$ are nonzero.

For an integer $s$ let $2^{R(s)}$ be the maximal power of 2 which divides $s$, and define $\kappa(n)=\max \left\{0<s<2^{n-1}: s-R(s)<2^{n-1}-n\right\}$. By using Corollary 3.2 (3) and following an argument similar to [At61, we obtain the following:

Proposition 3.4. For $n \geq 4, \mathbb{R} P^{2^{n}-1}$ does not admit tame corank one maps into $\mathbb{R}^{2^{n}-1-k}$ for $k \leq \kappa(n)-2$.

Remark 3.5. Obviously $s_{0}=2^{n-1}-2^{\min \left\{r: r+2^{r}>n\right\}}$ satisfies $s_{0}+n-R\left(s_{0}\right)<2^{n-1}$. Hence $s_{0} \leq \kappa(n)$, and we obtain that $\mathbb{R} P^{2^{n}-1}$ admits no tame corank one map into $\mathbb{R}^{2^{n-1}+2^{\min \left\{r: r+2^{r}>n\right\}}+j}$ for $n \geq 4$ and $j \geq 1$. Also, since $\min \left\{r: r+2^{r}>n\right\} \leq$ $\left\lceil\log _{2} n\right\rceil$, the same conclusion holds in the case of the target $\mathbb{R}^{2^{n-1}+2^{\left\lceil\log _{2} n\right\rceil}+j}$ for $n \geq 4$ and $j \geq 1$. For example, there exists neither a fold map from $\mathbb{R} P^{31}$ to $\mathbb{R}^{21+2 j}$ for $0 \leq j \leq 5$ nor a tame corank one map from $\mathbb{R} P^{31}$ to $\mathbb{R}^{22+2 j}$ for $0 \leq j \leq 4$.

\footnotetext{
${ }^{4}$ We presented these results at the conference "Singularity theory of smooth maps and related geometry", RIMS, Tokyo, and at the Topology Seminar at Kyushu University, Fukuoka, in December, 2009.

${ }^{5}$ Compare with At61 Proposition 3.2].

${ }^{6}$ We have $w\left(\mathbb{R} P^{2^{n}-1}\right)=(1+x)^{2^{n}}=1 \in \mathbb{Z}_{2}[x] / x^{2^{n}}=H^{*}\left(\mathbb{R} P^{2^{n}-1} ; \mathbb{Z}_{2}\right)$, where $x$ denotes the generator of $H^{1}\left(\mathbb{R} P^{2^{n}-1} ; \mathbb{Z}_{2}\right)$. The natural homomorphism $H^{s}\left(\mathbb{R} P^{2^{n}-1} ; \mathbb{Z}\right) \rightarrow H^{s}\left(\mathbb{R} P^{2^{n}-1} ; \mathbb{Z}_{2}\right)$ is an isomorphism for all positive even $s$. Our claim follows by applying the fact that $p_{i} \equiv w_{2 i}^{2}$ $\bmod 2$.
} 


\section{Computing the Characteristic Classes of the SOURCE MANifold}

Let $\gamma$ denote the line bundle over $\Sigma$ defined by the condition that $w_{1}(\gamma)$ is Poincaré dual to the class represented by $\Sigma^{k+1,1}$. We relate $\left.f^{*} d f(T M)\right|_{\Sigma}$ to $T \Sigma$ by the following.

Proposition 4.1. For a cusp map $f: M^{n} \rightarrow Q^{n-k}$, where $k$ is odd, we have $\left.T \Sigma \oplus \gamma \cong f^{*} d f(T M)\right|_{\Sigma} \oplus \varepsilon^{1}$.

Proof. Denote by $C$ the manifold $\Sigma^{k+1,1}$. Since $f$ is a cusp map, we have $C=$ $\Sigma^{k+1,1,0}$. We will first construct a bundle monomorphism

$$
i:\left.T \Sigma \rightarrow f^{*} d f(T M)\right|_{\Sigma} \oplus \varepsilon^{1}
$$

covering the identity map of $\Sigma$. Apart from $C$, the map $d f$ is an isomorphism between $T \Sigma$ and $\left.f^{*} d f(T M)\right|_{\Sigma}$. On $C$ the restriction of $d f$ to $T C$ is a monomorphism. Hence there is an isomorphism

$$
j:\left.\left.\left.T \Sigma\right|_{C} \rightarrow f^{*} d f(T C)\right|_{C} \oplus \operatorname{ker} d\left(\left.f\right|_{\Sigma}\right)\right|_{C}
$$

defined by composing the isomorphism $\left.\left.T \Sigma\right|_{C} \cong T C \oplus \operatorname{ker} d\left(\left.f\right|_{\Sigma}\right)\right|_{C}$ with the isomorphism

$$
\left(\left.d f\right|_{T C}, \operatorname{id}_{\left.\operatorname{ker} d\left(\left.f\right|_{\Sigma}\right)\right|_{C}}\right):\left.\left.\left.T C \oplus \operatorname{ker} d\left(\left.f\right|_{\Sigma}\right)\right|_{C} \rightarrow f^{*} d f(T C)\right|_{C} \oplus \operatorname{ker} d\left(\left.f\right|_{\Sigma}\right)\right|_{C} .
$$

Denote by $\operatorname{pr}_{\text {ker }}:\left.\left.T \Sigma\right|_{C} \rightarrow \operatorname{ker} d\left(\left.f\right|_{\Sigma}\right)\right|_{C}$ the composition of $j$ with the projection of $\left.f^{*} d f(T C) \oplus \operatorname{ker} d\left(\left.f\right|_{\Sigma}\right)\right|_{C}$ to the second factor.

Since ker $\left.d\left(\left.f\right|_{\Sigma}\right)\right|_{C} \subset T \Sigma$ is never tangent to $C$, we can identify it with the normal bundle of $C$ in $\Sigma$. But $k$ is odd hence this normal bundle is trivial - the indices of fold points on the two sides are different. After choosing a trivialization of ker $\left.d\left(\left.f\right|_{\Sigma}\right)\right|_{C}$, the map $j$ can be considered as an embedding of $\left.T \Sigma\right|_{C}$ into $\left.f^{*} d f(T M)\right|_{C} \oplus \varepsilon^{1}$, with its image $\operatorname{im} j=\left.f^{*} d f(T C)\right|_{C} \oplus \varepsilon^{1}$. This embedding extends as a fiberwise embedding onto a small neighborhood $N(C)$ of $C$ in $\Sigma$, and we will consider $j$ and also $\mathrm{pr}_{\text {ker }}$ to be defined on $N(C)$.

Define $i$ to be the linear interpolation of the embedding

$$
j:\left.\left.T \Sigma\right|_{N(C)} \rightarrow f^{*} d f(T M)\right|_{N(C)} \oplus \varepsilon^{1}
$$

and the map

$$
\left(\left.d f\right|_{T \Sigma}, 0\right):\left.T \Sigma \rightarrow f^{*} d f(T M)\right|_{\Sigma} \oplus \varepsilon^{1} .
$$

That is, we take a bump function $\lambda: T \Sigma \rightarrow[0,1]$ such that $\lambda=0$ outside a tubular neighborhood of $C$ in $\Sigma$ and $\lambda^{-1}(\{1\})=\left.T \Sigma\right|_{C}$, and we define $i$ to be $\left(\left.d f\right|_{T \Sigma}, \lambda \mathrm{pr}_{\mathrm{ker}}\right):\left.T \Sigma \rightarrow f^{*} d f(T M)\right|_{\Sigma} \oplus \varepsilon^{1}$. Thus $i$ is well-defined, since $\lambda=0$ where $\mathrm{pr}_{\mathrm{ker}}$ is not defined, and it is clear that $i$ has full rank both on $C$ and its complement in $\Sigma$.

From this embedding $i$, we get $\left.f^{*} d f(T M)\right|_{\Sigma} \oplus \varepsilon^{1} \cong T \Sigma \oplus$ coker $i$, and we only need to identify coker $i$ with $\gamma$. Indeed, on the set $\Sigma-C$ the line bundle coker $i$ is trivial as $\operatorname{im} i$ projects isomorphically onto $\left.f^{*} d f(T M)\right|_{\Sigma}$. On a tubular neighborhood of $C$, this trivialization of coker $\left.i\right|_{\Sigma-C}$ has the opposite signs on the two sides of $C$. Thus $w_{1}(\operatorname{coker} i)$ is dual to $C$ in $\Sigma$, as claimed.

Corollary 4.2. For a cusp map $f: M^{n} \rightarrow Q^{n-k}$, where $k$ is odd, we have

$$
w(\Sigma)=w\left(\left.f\right|_{\Sigma}{ }^{*} T Q\right) w(\nu)^{-1} w(\gamma)^{-1},
$$

where $\nu$ denotes the line bundle $\left.f^{*} T Q\right|_{\Sigma} /\left.f^{*} d f(T M)\right|_{\Sigma}$. 
Let $c \in H^{1}(\Sigma)$ denote the characteristic class $w_{1}(\gamma)$. As noted above, the manifold $\Sigma^{k+1,1}$ has a trivial normal bundle in $\Sigma$, thus $c^{2}=0$. Let $b$ denote $w_{1}\left(\left.f^{*} T Q\right|_{\Sigma} /\left.f^{*} d f(T M)\right|_{\Sigma}\right) \in H^{1}(\Sigma)$.

We will use the notation of the following blowup diagram:

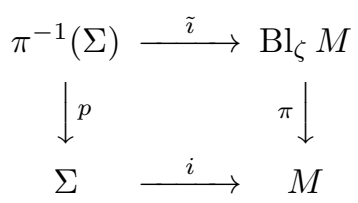

Proof of Theorem 1.1. Let $\delta$ be 0 if $f$ is a fold map, and let $\delta$ be 1 otherwise. If the Morin map $f: M^{n} \rightarrow Q^{n-k}$ is not a cusp map and both $M$ and $Q$ are orientable, then perturb $f$ to get a cusp map (see [Sad03]) and denote this cusp map also by $f$ for simplicity.

Following the blowup formula for Stiefel-Whitney classes GP07, Theorem 10 and Remark (2) on page 328], we can express in our notation the Stiefel-Whitney classes of $T M$ in terms of those of $T \mathrm{Bl}_{\xi} M, T \Sigma$ and $\xi$ in the following way:

$$
\begin{aligned}
w\left(\mathrm{Bl}_{\xi} M\right)- & w\left(\pi^{*} T M\right) \\
= & \tilde{\imath}_{!}\left(w\left(p^{*} T \Sigma\right) \frac{1}{w_{1}(\mu)}\left(\sum_{t=0}^{k+1} w_{t}\left(p^{*} \xi\right)\left(1+w_{1}(\mu)\right)^{k+2-t}-w\left(p^{*} \xi\right)\right)\right) .
\end{aligned}
$$

Here $\mu$ denotes the canonical line bundle over $\pi^{-1}(\Sigma)$ (which is just the canonical line bundle over each fiber). Recall that $p$ is the restriction $\left.\pi\right|_{\pi^{-1}(\Sigma)}$ and that the map $\tilde{\imath}: \pi^{-1}(\Sigma) \rightarrow \mathrm{Bl}_{\xi} M$ is the natural embedding. By Theorems 2.6 and 2.7. the total Stiefel-Whitney class of $T \mathrm{Bl}_{\xi} M$ is equal to the product of the total Stiefel-Whitney class of a $(k+1+\delta)$-dimensional vector bundle and the total Stiefel-Whitney class of $\pi^{*} f^{*} T Q$, which contains no term of degree greater than $K$. Therefore $w_{l}\left(\mathrm{Bl}_{\xi} M\right)=0$ for $l>k+K+1+\delta$.

Expanding the blowup formula for $r \geq k+K+2+\delta$ we thus get

$$
\begin{aligned}
& w_{r}\left(\pi^{*} T M\right)=w_{r}\left(\mathrm{Bl}_{\xi} M\right) \\
& +\tilde{\imath}_{!}\left(\sum_{q=0}^{r-1} w_{r-1-q}\left(p^{*} T \Sigma\right)\left[\sum_{t=0}^{k+1} w_{t}\left(p^{*} \xi\right) \sum_{s=0}^{k+1-t}\left(\begin{array}{c}
k+2-t \\
s+1
\end{array}\right) w_{1}(\mu)^{s}\right]_{\mathrm{deg}=q}\right) \\
& =\tilde{\imath}_{!}\left(\sum_{q=0}^{k+1} p^{*} w_{r-1-q}(\Sigma) \sum_{t=0}^{k+1}\left(\begin{array}{c}
k+2-t \\
q-t+1
\end{array}\right) p^{*} w_{t}(\xi) w_{1}(\mu)^{q-t}\right),
\end{aligned}
$$

where we use our convention about binomial coefficients and $w_{1}(\mu)^{-1}$ is defined to be 0 .

The classes $w_{r-1-q}(\Sigma)$ can be obtained from Corollary 4.2, Under the assumption that $m \geq K+\delta$, we have

$$
\begin{aligned}
w_{m}(\Sigma) & =\left[w\left(\left.f\right|_{\Sigma}{ }^{*} T Q\right) w(\nu)^{-1} w(\gamma)^{-1}\right]_{\mathrm{deg}=m} \\
& =\left.\sum_{l=0}^{m} f\right|_{\Sigma}{ }^{*} w_{l}(Q)\left(b^{m-l}+b^{m-l-1} c\right)=\left.\sum_{l=0}^{K} f\right|_{\Sigma}{ }^{*} w_{l}(Q)\left(b^{m-l}+b^{m-l-1} c\right) .
\end{aligned}
$$


Notice that in the formula all the exponents of $b$ are at least 0 . Substituting $m=r-1-q$, where $0 \leq q \leq k+1$, we get that

$$
w_{r-1-q}(\Sigma)=b^{r-1-q-K-\delta} w_{K+\delta}(\Sigma)
$$

for all $0 \leq q \leq k+1$. Hence we have that if $r \geq k+K+2+\delta$, then

$$
\begin{aligned}
& w_{r}\left(\pi^{*} T M\right) \\
& =\tilde{\imath}_{!}\left(\sum_{q=0}^{k+1} p^{*}\left(b^{r-1-q-K-\delta} w_{K+\delta}(\Sigma)\right) \sum_{t=0}^{k+1}\left(\begin{array}{c}
k+2-t \\
q-t+1
\end{array}\right) p^{*} w_{t}(\xi) w_{1}(\mu)^{q-t}\right) \\
& =\tilde{\imath}_{!}\left(p^{*} b^{r-k-K-2-\delta} \sum_{q=0}^{k+1} \sum_{t=0}^{k+1}\left(\begin{array}{c}
k+2-t \\
q-t+1
\end{array}\right) p^{*}\left(b^{k+1-q} w_{K+\delta}(\Sigma) w_{t}(\xi)\right) w_{1}(\mu)^{q-t}\right) .
\end{aligned}
$$

Notice that the double sum in this formula does not depend on $r$ at all, and let $\alpha$ denote

$$
\sum_{q=0}^{k+1} \sum_{t=0}^{k+1}\left(\begin{array}{c}
k+2-t \\
q-t+1
\end{array}\right) p^{*}\left(b^{k+1-q} w_{K+\delta}(\Sigma) w_{t}(\xi)\right) w_{1}(\mu)^{q-t}
$$

Then

$$
w_{r}\left(\pi^{*} T M\right)=\tilde{\imath}_{!}\left(\alpha p^{*} b^{r-k-K-2-\delta}\right)
$$

holds for all $r \geq k+K+2+\delta$, and we can calculate products of these characteristic classes by repeatedly applying the formula $\tilde{\imath}_{!}(u) \tilde{\imath}_{!}(u)=\tilde{\imath}_{!}(1) \tilde{\imath}_{!}(u v)$ as follows. For $r_{1}, \ldots, r_{m} \geq k+K+2+\delta$, we have

$$
\begin{aligned}
& w_{r_{1}}\left(\pi^{*} T M\right) \ldots w_{r_{m}}\left(\pi^{*} T M\right)=\prod_{i=1}^{m} \tilde{\imath}_{!}\left(\alpha p^{*} b^{r_{i}-k-K-2-\delta}\right) \\
& =\tilde{\imath}_{!}(1)^{m-1} \tilde{\imath}_{!}\left(\prod_{i=1}^{m} \alpha p^{*} b^{r_{i}-k-K-2-\delta}\right)=\tilde{\imath}_{!}(1)^{m-1} \tilde{\imath}_{!}\left(\alpha^{m} p^{*} b^{\sum_{i=1}^{m}\left(r_{i}-k-K-2-\delta\right)}\right) .
\end{aligned}
$$

This expression clearly depends only on $m$ and the sum $r_{1}+\cdots+r_{m}$, and since the homomorphism $\pi^{*}: H^{*}\left(M ; \mathbb{Z}_{2}\right) \rightarrow H^{*}\left(\mathrm{Bl}_{\xi} M ; \mathbb{Z}_{2}\right)$ is injective, this proves the statement of the theorem.

Lemma 4.3. Let $n=2^{D}+m, 0 \leq m<2^{D}$. Then $\left(\begin{array}{l}n \\ m\end{array}\right)$ is odd, and $\left(\begin{array}{l}n \\ r\end{array}\right)$ is even if $m<r<2^{D}$.

Proof. A criterion of [G199] states that $\left(\begin{array}{l}b \\ a\end{array}\right), 0 \leq a \leq b$, is even if and only if there is a binary position at which $a$ has the digit 1 and $b$ has the digit 0 . This criterion shows that $\left(\begin{array}{l}n \\ m\end{array}\right)$ is odd. If $\left(\begin{array}{l}n \\ r\end{array}\right)$ is odd for some $0 \leq r<2^{D}$, then all the binary digits of $r$ at the positions where $n$ has 0 have to be 0 as well. Since $r$ has binary length at most $D$, this is equivalent to the condition that $r$ has binary digits 1 only at positions where $m=n-2^{D}$ has 1 as well, hence the maximal such $r$ is $m$, as claimed.

Proposition 4.4. Let $n=2^{D}+m$ with $0 \leq m<2^{D}-2$. Assume there exists an integer $l$ such that the equations $w_{a} w_{b}\left(\mathbb{R} P^{n}\right)=w_{c} w_{d}\left(\mathbb{R} P^{n}\right)$ hold for all $a, b, c, d \geq l$. Then $l \geq m+1$. 
Proof. Denote the generator of $H^{1}\left(\mathbb{R} P^{n} ; \mathbb{Z}_{2}\right)$ by $x$. Then we have

$$
H^{*}\left(\mathbb{R} P^{n} ; \mathbb{Z}_{2}\right)=\mathbb{Z}_{2}[x] /\left(x^{n+1}\right)
$$

and

$$
w_{j}\left(\mathbb{R} P^{n}\right)=\left(\begin{array}{c}
n+1 \\
j
\end{array}\right) x^{j} .
$$

By Lemma $4.3\left(\begin{array}{c}n+1 \\ m+1\end{array}\right)=\left(\begin{array}{c}n \\ m\end{array}\right)+\left(\begin{array}{c}n \\ m+1\end{array}\right)$ is odd, hence the class $w_{m+1}\left(\mathbb{R} P^{n}\right)$ is the generator $x^{m+1}$, while the classes $w_{m+2}\left(\mathbb{R} P^{n}\right), \ldots, w_{2^{D}-1}\left(\mathbb{R} P^{n}\right)$ vanish. Note that there is at least one class in this latter list due to the constraint $m<2^{D}-2$. In particular, the class $w_{m} w_{m+2}\left(\mathbb{R} P^{n}\right)$ also has to vanish, while $w_{m+1}^{2}\left(\mathbb{R} P^{n}\right)=x^{2 m+2}$ is not zero as $2 m+2<2^{D}-2+m+2=n<n+1$. Therefore the relation $w_{m+1}^{2}=w_{m+2} w_{m}$ does not hold on $\mathbb{R} P^{n}$, implying $l>m$.

Proposition 4.5. Let $n=2^{D}+m$ with $0 \leq m<2^{D}-2$. Then the relations

$$
\prod_{i \in I} w_{i}\left(\mathbb{R} P^{n}\right)=\prod_{j \in J} w_{j}\left(\mathbb{R} P^{n}\right)
$$

hold for all $I, J \subseteq\{0, \ldots, n\}$ which satisfy $|I|=|J|, \min I, \min J \geq m+1$ and $\sum_{i \in I} i=\sum_{j \in J} j$.

Proof. As before, we note that the classes $w_{m+2}\left(\mathbb{R} P^{n}\right), \ldots, w_{2^{D}-1}\left(\mathbb{R} P^{n}\right)$ vanish.

For $|I|=1$ the statement is trivial. For $|I| \geq 2$ such that $\min I \geq m+1$, we have three possibilities:

- $I$ consists of a number of copies of $m+1$. Then the only $J$ which satisfies both $\min J \geq m+1$ and $\sum_{j \in J} j=\sum_{i \in I} i=(m+1)|I|$ is $I$ itself.

- $I$ contains an index between $m+2$ and $2^{D}-1$. Then $\prod_{i \in I} w_{i}\left(\mathbb{R} P^{n}\right)$ contains a zero class and thus vanishes.

- I contains at least one index greater than $2^{D}-1$. Then taking any other index $j \in I$ we have $\sum_{i \in I} i \geq 2^{D}+j \geq 2^{D}+m+1=n+1$. Therefore $\prod_{i \in I} w_{i}\left(\mathbb{R} P^{n}\right)$ has degree greater than $n$ and consequently vanishes.

Observe that for any $J$ satisfying the requirements of the proposition we have the analogous three possibilities. Hence any such $J$ gives the same product of StiefelWhitney classes as $I$.

Remark 4.6. In the cases $n=2^{D}-2$ and $n=2^{D}-1$ the nontrivial characteristic classes of $\mathbb{R} P^{n}$ are either all the generators of the respective cohomology groups $H^{*}\left(\mathbb{R} P^{n}\right)$ or all vanish. Therefore our multiplicativity condition is satisfied for all indices.

Proof of Theorem 1.3. Follows from Theorem 1.1 and the propositions above; details are left to the reader.

Remark 4.7. By [MS74, Corollary 11.15], we obtain the result analogous to Theorem 1.3 for closed $n$-manifolds $M$ with $H^{*}\left(M ; \mathbb{Z}_{2}\right) \cong H^{*}\left(\mathbb{R} P^{n} ; \mathbb{Z}_{2}\right)$. Also a more general result for any closed manifold whose cohomology ring with $\mathbb{Z}_{2}$ coefficients is generated by one element.

Proof of Theorem 1.4. Equip $T M$ and $T Q$ with Riemannian metrics, thus identifying sections of these bundles with 1-forms. Assume that we have a trivialization 
of $T Q \oplus \varepsilon^{l}$ given by a collection of $n-k+l$ linearly independent 1 -forms. Then any smooth map $f: M \rightarrow Q$ defines pullbacks of these 1-forms to $T M \oplus \varepsilon^{l}$ via $d f$. By the assumption of the theorem, rank $d f \geq n-k-1$ at all points of $M$. Thus the linear span of the pulled-back forms is at least $n-k+l-1$. The metric on $T M$ identifies these forms with $n-k+l$ vector fields which have a linear span of dimension at least $n-k+l-1$ everywhere. By Roh52, Ga78, the rational Pontryagin class $p_{i}^{\mathbb{Q}}(M)=p_{i}^{\mathbb{Q}}\left(M \oplus \varepsilon^{l}\right)$ is represented by the locus where $n+l-2 i+2$ generic sections of $T M \oplus \varepsilon^{l}$ lie in a subspace of dimension at most $n+l-2 i$. This class therefore vanishes if $n-k+l \geq n+l-2 i+2$, that is, when $2 i \geq k+2$.

Proof of Proposition 3.1. The equivalence (1) $\Longleftrightarrow(2)$ follows from an argument similar to [An04, Proof of Lemma 3.1].

If (1) or (2) holds and $Q$ is stably parallelizable, then by the above, we have $T M \oplus \varepsilon^{1} \oplus \varepsilon^{N} \cong \zeta^{k+1} \oplus f^{*} T Q \oplus \varepsilon^{N} \cong \zeta^{k+1} \oplus \varepsilon^{N+n-k}$ for some $N \gg 0$ and a $(k+1)$-dimensional bundle $\zeta^{k+1}$. Thus g.dim $\left([T M]-\left[\varepsilon^{n}\right]\right) \leq k+1$.

If $Q$ is stably parallelizable and $\operatorname{g\cdot dim}\left([T M]-\left[\varepsilon^{n}\right]\right) \leq k+1$, then $T M \oplus \varepsilon^{N} \cong$ $\zeta^{k+1} \oplus \varepsilon^{N+n-k-1} \cong \zeta^{k+1} \oplus f^{*} T Q \oplus \varepsilon^{N-1}$ for some $N \gg 0$, and thus $T M \oplus \varepsilon^{1} \cong$ $\zeta^{k+1} \oplus f^{*} T Q$, which proves $(2)$.

Remark 4.8. If there is a tame Morse-Bott map $f: P^{n} \rightarrow Q^{n-k}$, then $T P \oplus \varepsilon^{1}$ splits as $\zeta^{k+1} \oplus f^{*} T Q$ for some $(k+1)$-dimensional vector bundle $\zeta^{k+1}$.

Proof of Proposition 3.4. Let $\varphi(n)$ denote the cardinality of the set $\{0<s \leq n$ : $s \equiv 0,1,2,4 \bmod 8\}$. By $[$ At61, $\S 5],\left[T \mathbb{R} P^{n}\right]-\left[\varepsilon^{n}\right]=(n+1) x$ and

$$
\gamma^{i}\left(\left[T \mathbb{R} P^{n}\right]-\left[\varepsilon^{n}\right]\right)=2^{i-1}\left(\begin{array}{c}
n+1 \\
i
\end{array}\right) x, \quad i \geq 1
$$

where $x$ denotes the generator of $\widetilde{K}_{\mathbb{R}}\left(\mathbb{R} P^{n}\right)=\mathbb{Z}_{2 \varphi(n)}$. Therefore $\gamma^{i}\left(\left[T \mathbb{R} P^{n}\right]-\left[\varepsilon^{n}\right]\right)=$ 0 if and only if $2^{\varphi(n)}$ divides $2^{i-1}\left(\begin{array}{c}n+1 \\ i\end{array}\right)$. Let $r(n)$ denote the greatest integer $s$ for which $2^{s-1}\left(\begin{array}{c}n+1 \\ s\end{array}\right)$ is not divisible by $2^{\varphi(n)}$. Then by Proposition 3.1 there is no tame corank one map of $\mathbb{R} P^{2^{n}-1}$ into $\mathbb{R}^{2^{n}-1-k}$ for $k \leq r\left(2^{n}-1\right)-2$. It is easy to see that $\varphi\left(2^{n}-1\right)=2^{n-1}-1$ if $n \geq 3$. By a classical result of E. Kummer, the highest power $c(s)$ of 2 which divides $\left(\begin{array}{c}2^{n} \\ s\end{array}\right)$ can be obtained by counting the number of carries when $s$ and $2^{n}-s$ are added in base 2. For $s \leq 2^{n-1}-1$, we claim that $c(s)=n-R(s)$, where $2^{R(s)}$ is the maximal power of 2 which divides $s$. Indeed, $2^{n}-1-s$ is obtained by negating the binary form of $s$ bitwise. Hence $2^{n}-s$ is obtained by negating the binary form of $s$ bitwise from the $(n-1)$-st to the $R(s)$-th binary position, where both of $s$ and $2^{n}-s$ have the digit 1 , and after that position both have digits 0 . Therefore when we add $s$ and $2^{n}-s$ in base 2 , we have $n-R(s)$ carries. By the definition of $r(n)$ it follows that $r\left(2^{n}-1\right)$ is the largest integer $s$ for which $s+n-R(s)<2^{n-1}$.

4.1. Computing the cobordism class of the source manifold. Theorem 1.1 gives us relations among the characteristic numbers of a source manifold of a Morin map as well. However, by following a different line of argument, we can obtain more relations among the characteristic numbers as follows.

For a Morin map $f: M^{n} \rightarrow Q^{n-k}$ with odd $k \geq 1$, let us denote by $N_{\Sigma}$ the projectivization $\mathbb{R} P\left(\xi \oplus \varepsilon^{1}\right)$ of the $(k+2)$-dimensional vector bundle $\xi \oplus \varepsilon^{1}$ over 
the singularity set $\Sigma$, where $\xi$ denotes the normal bundle of $\Sigma$. Thus $N_{\Sigma}$ is a closed $n$-dimensional manifold fibered over $\Sigma$ with $\mathbb{R} P^{k+1}$ as fiber. Let $\tau: N_{\Sigma} \rightarrow \Sigma$ denote this fibration.

Lemma 4.9. The blowup $\mathrm{Bl}_{\xi} M$ is cobordant to the disjoint union of $M$ and $N_{\Sigma}$.

Proof. Consider the disk bundle $D\left(\xi \oplus \varepsilon^{1}\right)$ of $\xi \oplus \varepsilon^{1}$. Let $U$ and $V$ be small neighborhoods of $\xi \oplus\{1\}$ and $\xi \oplus\{-1\}$ respectively in the boundary $\partial D\left(\xi \oplus \varepsilon^{1}\right)$. The total space of $D\left(\xi \oplus \varepsilon^{1}\right)$ can be naturally glued to the boundary component $\left(M \sqcup \mathbb{R} P\left(\xi \oplus \varepsilon^{1}\right)\right) \times\{0\}$ of $\left(M \sqcup \mathbb{R} P\left(\xi \oplus \varepsilon^{1}\right)\right) \times[0,1]$ along $U$ identified with the total space of $\xi$ as an open submanifold of $M$ and along $V$ identified with $[\xi: 1] \subset \mathbb{R} P\left(\xi \oplus \varepsilon^{1}\right)$. After smoothing the corners introduced by the gluing, the resulting $(n+1)$-manifold has boundary consisting of the disjoint union of $M$, $\mathbb{R} P\left(\xi \oplus \varepsilon^{1}\right)$ and $\mathrm{Bl}_{\xi} M$. This completes the proof.

Remark 4.10. As one can easily see, the cobordism in Lemma4.9 extends naturally to a bordism of the maps $\mathrm{Bl}_{\xi} f: \mathrm{Bl}_{\xi} M \rightarrow Q$ and the union $\left.f \sqcup f\right|_{\Sigma} \circ \tau: M \sqcup$ $N_{\Sigma} \rightarrow Q$. Indeed, we can map all points of each fiber of $D\left(\xi \oplus \varepsilon^{1}\right)$ over $p$ to $p$, where $p \in \Sigma$, and then into $Q$ by $\left.f\right|_{\Sigma}$. Thus the evaluation of any "characteristic number" $w_{I}$ (i.e. degree $n$ monomial of Stiefel-Whitney characteristic classes) of $T \mathrm{Bl}_{\xi} M-\left(\mathrm{Bl}_{\xi} f\right)^{*} T Q$ on the fundamental class $\left[\mathrm{Bl}_{\xi} M\right] \in H_{n}\left(\mathrm{Bl}_{\xi} M ; \mathbb{Z}_{2}\right)$ is equal to the sum of the evaluations of $w_{I}\left(T M-f^{*} T Q\right)$ and $w_{I}\left(T N_{\Sigma}-\left.\tau^{*} f\right|_{\Sigma} ^{*} T Q\right)$ on the fundamental classes $[M]$ and $\left[N_{\Sigma}\right]$, respectively.

Recall that $b$ denotes $w_{1}\left(\left.f^{*} T Q\right|_{\Sigma} /\left.f^{*} d f(T M)\right|_{\Sigma}\right) \in H^{1}(\Sigma)$ and $w_{1}(\gamma)=c \in$ $H^{1}(\Sigma)$ is the Poincaré dual to the class represented by $\Sigma^{k+1,1}$. We have also seen that $c^{2}=0$.

Proposition 4.11. Let $f: M^{n} \rightarrow Q^{n-k}$ be a cusp map. Let $\delta$ be 0 if $f$ is a fold map, and let $\delta$ be 1 otherwise.

(1) For $r \geq k+1+\delta$, the class

$$
w_{r}\left(T N_{\Sigma}-\left.\tau^{*} f\right|_{\Sigma}{ }^{*} T Q\right)=\tau^{*} b^{r-k-1-\delta} w_{k+1+\delta}\left(T N_{\Sigma}-\left.\tau^{*} f\right|_{\Sigma}{ }^{*} T Q\right)
$$

(2) Any two characteristic numbers of $w\left(T N_{\Sigma}-\left.\tau^{*} f\right|_{\Sigma}{ }^{*} T Q\right)$ which contain the same number of multiplicands and contain no instances of $w_{1}, \ldots, w_{k+\delta}$ are equal.

(3) For any multiindex $J=\left(j_{1}, \ldots, j_{l}\right)$ such that $\sum_{i=0}^{l} j_{i}=n$ and $j_{i} \geq k+2+\delta$ for some $1 \leq i \leq l$, the characteristic numbers $\left\langle w_{J}\left(T M-f^{*} T Q\right),[M]\right\rangle$ and $\left\langle w_{J}\left(T N_{\Sigma}-\left.\tau^{*} f\right|_{\Sigma}{ }^{*} T Q\right),\left[N_{\Sigma}\right]\right\rangle$ coincide. The characteristic numbers defined by $w\left(T M-f^{*} T Q\right)$ which do not involve any of the classes $w_{1}, \ldots, w_{k+\delta}$ satisfy the property of depending only on the number of multiplicands.

Proof. The fibration $\tau: N_{\Sigma} \rightarrow \Sigma$ has fiber $\mathbb{R} P^{k+1}$, and $T N_{\Sigma}$ splits into the direct sum of the horizontal component $\tau^{*} T \Sigma$ and the vertical component $\psi$ having rank $k+1$, which is tangent to the fibers. Recall that $\nu$ denotes the line bundle $\left.f^{*} T Q\right|_{\Sigma} /\left.f^{*} d f(T M)\right|_{\Sigma}$. By Corollary 4.2, we have

$$
w\left(\left.f\right|_{\Sigma}{ }^{*} T Q\right)^{-1} w(\Sigma)=w(\nu)^{-1} w(\gamma)^{-1}=(1+b)^{-1}(1+c)^{-1}=\sum_{i=0}^{\infty} b^{i}(1+c) .
$$


Hence we can express $w\left(\left.\tau^{*} f\right|_{\Sigma}{ }^{*} T Q\right)^{-1} w\left(N_{\Sigma}\right)$ as

$$
\begin{array}{rl}
w\left(\left.\tau^{*} f\right|_{\Sigma}{ }^{*} T Q\right)^{-1} & w\left(N_{\Sigma}\right)=w\left(\left.\tau^{*} f\right|_{\Sigma}{ }^{*} T Q\right)^{-1} \tau^{*} w(\Sigma) w(\psi) \\
=\left(1+\sum_{i=1}^{\infty}\left(\tau^{*} b^{i}+\tau^{*} b^{i-1} c\right)\right)\left(\sum_{j=0}^{k+1} w_{j}(\psi)\right) \\
\stackrel{(\mathrm{a})}{=} \sum_{r=0}^{\infty} \sum_{j=0}^{\min \{r, k+1\}} w_{j}(\psi)\left(\tau^{*} b^{r-j}+\tau^{*} b^{r-j-1} c\right),
\end{array}
$$

where (a) follows from rearranging the sums by $i+j=r$, and we use the convention that $b^{-1}=0$. The class $w_{r}\left(T N_{\Sigma}-\left.\tau^{*} f\right|_{\Sigma}{ }^{*} T Q\right)$ in the case of $r \geq k+2$ therefore has the form

$$
\begin{aligned}
w_{r}\left(T N_{\Sigma}-\left.\tau^{*} f\right|_{\Sigma}{ }^{*} T Q\right) & =\sum_{j=0}^{k+1} w_{j}(\psi)\left(\tau^{*} b^{r-j}+\tau^{*} b^{r-j-1} c\right) \\
& =\tau^{*} b^{r-k-2} w_{k+2}\left(T N_{\Sigma}-\left.\tau^{*} f\right|_{\Sigma}{ }^{*} T Q\right)
\end{aligned}
$$

If additionally $\delta=0$ (thus $c=0$ ), then similarly we have

$$
w_{r}\left(T N_{\Sigma}-\left.\tau^{*} f\right|_{\Sigma}{ }^{*} T Q\right)=\sum_{j=0}^{k+1} w_{j}(\psi) \tau^{*} b^{r-j}=\tau^{*} b^{r-k-1} w_{k+1}\left(T N_{\Sigma}-\left.\tau^{*} f\right|_{\Sigma}{ }^{*} T Q\right)
$$

for $r \geq k+1$. These two equalities prove (1).

Now consider a product $\prod_{i=1}^{m} w_{j_{i}}\left(T N_{\Sigma}-\left.\tau^{*} f\right|_{\Sigma}{ }^{*} T Q\right)$ which contains no instances of $w_{1}\left(T N_{\Sigma}-\left.\tau^{*} f\right|_{\Sigma}{ }^{*} T Q\right), \ldots, w_{k+\delta}\left(T N_{\Sigma}-\left.\tau^{*} f\right|_{\Sigma}{ }^{*} T Q\right)$. By (1), it has the form

$$
\tau^{*} b^{n-(k+1+\delta) m} w_{k+1+\delta}^{m}\left(T N_{\Sigma}-\left.\tau^{*} f\right|_{\Sigma}{ }^{*} T Q\right) .
$$

In particular, this expression depends only on $m$, and hence any two characteristic numbers of the virtual bundle $T N_{\Sigma}-\left.\tau^{*} f\right|_{\Sigma}{ }^{*} T Q$ containing the same number of multiplicands and no instances of $w_{1}, \ldots, w_{k+\delta}$ are equal. This proves (2).

By Theorems 2.6 and 2.7 the formal difference bundle $T \mathrm{Bl}_{\xi} M-\pi^{*} f^{*} T Q$ is stably equivalent to a $(k+1+\delta)$-dimensional bundle. Therefore the characteristic classes $w_{r}\left(T \mathrm{Bl}_{\xi} M-\pi^{*} f^{*} T Q\right)$ of $T \mathrm{Bl}_{\xi} M-\pi^{*} f^{*} T Q$ with $r>k+1+\delta$ vanish. Thus, by Remark 4.10 those characteristic numbers of the virtual normal bundles of the maps $N_{\Sigma} \rightarrow Q$ and $M \rightarrow Q$ which contain $w_{r}$ with $r>k+1+\delta$ coincide. This finishes the proof of (3).

Corollary 4.12. Let $M$ be $k$-orientable (i.e. $w_{i}(M)=0$ for $1 \leq i \leq k$ ) and let $Q$ be stably parallelizable. If there exists a fold map $M^{n} \rightarrow Q^{n-k}$, then each of the nonzero characteristic numbers of $M$, which has more than one multiplicand, is equal to a number of the form $w_{k+1}^{l} w_{n-(k+1) l}[M]$ with $0 \leq l \leq \frac{n}{k+1}-1$.

4.1.1. Adding Dold relations to the relations of Proposition 4.11, In this section, we work in the case of fixed $n \geq 2, k=1$ and assume an orientable source manifold $M^{n}$. 
Denote by $\mathcal{I}$ the linear space in the graded $\mathbb{Z}_{2}$-algebra $\mathbb{Z}_{2}\left[w_{1}, w_{2}, \ldots\right]$ spanned by the set

$$
\begin{aligned}
& \left\{q_{1}-q_{2} \in \mathbb{Z}_{2}\left[w_{1}, \ldots, w_{n}\right]_{\operatorname{deg}=n}: q_{1}, q_{2} \text { are monomials in } \mathbb{Z}_{2}\left[w_{2}, \ldots, w_{n}\right]_{\operatorname{deg}=n},\right. \\
& \left.\left|q_{1}\right|=\left|q_{2}\right|\right\} \cup\left\{q_{1} \in\left[w_{1} \mathbb{Z}_{2}\left[w_{1}, \ldots, w_{n}\right]\right]_{\operatorname{deg}=n}\right\},
\end{aligned}
$$

where $|q|$ denotes the length of the monomial $q$, i.e. the number of (not necessarily different) variables whose product is $q$. Proposition 4.11 (3) states that if $M$ is an $n$-manifold admitting a codimension -1 fold map into a stably parallelizable target, then the evaluation $w_{i}=w_{i}(M)$ sends all the members of $\mathcal{I}$ to 0 .

Denote by $\mathcal{D}$ the linear space spanned in $\mathbb{Z}_{2}\left[w_{1}, \ldots, w_{n}, \ldots\right]$ by the set

$$
\left\{q \in \mathbb{Z}_{2}\left[w_{1}, \ldots, w_{n}\right]: p \in \mathbb{Z}_{2}\left[w_{1}, \ldots, w_{n}, \ldots\right]_{\operatorname{deg} \leq n-1}, q=\left[w^{-1} \cdot \operatorname{Sq} p\right]_{\operatorname{deg}=n}\right\},
$$

where $w$ stands for the total Stiefel-Whitney class $1+w_{1}+\cdots+w_{n}+\cdots$. We will apply a result of Dold Do56, which states that all the relations between the characteristic numbers of $n$-manifolds are exactly those of the form $q=0$ for $q \in \mathcal{D}$. Combining this set of relations with $\{q=0: q \in \mathcal{I}\}$ and proving that $\operatorname{dim} \mathbb{Z}_{2}\left[w_{1}, \ldots, w_{n}\right]_{\operatorname{deg}=n} /(\mathcal{D} \oplus \mathcal{I})=0$ unless $n=2^{a}+2^{b}-1$ for some $a>b \geq 0$ forms the core of the proof of Theorem 1.6 .

To utilize the relations obtained in Proposition 4.11 (3), we will consider the graded algebra homomorphism

$$
\begin{gathered}
\varrho: \mathbb{Z}_{2}\left[w_{1}, \ldots, w_{n}, \ldots\right] \rightarrow \mathbb{Z}_{2}[x, t], \operatorname{deg} x=2, \operatorname{deg} t=1, \\
\varrho\left(w_{1}\right)=0, \varrho\left(w_{s}\right)=x t^{s-2} \text { for } s \geq 2 .
\end{gathered}
$$

Define im $\varrho_{n}$ to be $\mathbb{Z}_{2}[x, t]_{\operatorname{deg}=n} \cap \operatorname{im} \varrho=\left\langle x t^{n-2}, \ldots, x^{\lfloor n / 2\rfloor} t^{n-2\lfloor n / 2\rfloor}\right\rangle$. It is straightforward to see that ker $\varrho \cap \mathbb{Z}_{2}\left[w_{1}, \ldots\right]_{\operatorname{deg}=n}$ is exactly $\mathcal{I}$. Therefore

$$
\begin{aligned}
& \operatorname{dim} \mathbb{Z}_{2}\left[w_{1}, \ldots\right]_{\operatorname{deg}=n} /(\mathcal{D} \oplus \mathcal{I})=\operatorname{dim}\left(\mathbb{Z}_{2}\left[w_{1}, \ldots\right]_{\operatorname{deg}=n} / \mathcal{I}\right) /(\mathcal{D} / \mathcal{D} \cap \mathcal{I}) \\
& \quad=\operatorname{dim}\left(\mathbb{Z}_{2}\left[w_{1}, \ldots\right]_{\operatorname{deg}=n} / \mathcal{I}\right)-\left.\operatorname{dimim} \varrho\right|_{\mathcal{D}}=\operatorname{dim} \operatorname{im} \varrho_{n}-\left.\operatorname{dimim} \varrho\right|_{\mathcal{D}}
\end{aligned}
$$

To calculate the image of $\mathcal{D}$ under $\varrho$, we use the Wu formulas. For $u \geq 2$

$$
\begin{aligned}
& \varrho \circ \mathrm{Sq} w_{u}=\varrho\left(\sum_{d=0}^{u} \sum_{j=0}^{d}\left(\begin{array}{c}
u-d+j-1 \\
j
\end{array}\right) w_{u+j} w_{d-j}\right) \\
& =\sum_{d=0}^{u}\left(\left(\begin{array}{c}
u-1 \\
d
\end{array}\right) x t^{u+d-2}+\sum_{j=0}^{d-2}\left(\begin{array}{c}
u-d+j-1 \\
j
\end{array}\right) x^{2} t^{u+d-4}\right) \\
& =\sum_{d=0}^{u}\left(\left(\begin{array}{c}
u-1 \\
d
\end{array}\right) x t^{u+d-2}+\left(\begin{array}{c}
u-2 \\
d-2
\end{array}\right) x^{2} t^{u+d-4}\right) \\
& =x t^{u-2}(t+1)^{u-1}+x^{2} t^{u-2}(t+1)^{u-2}=x t^{u-2}(t+1)^{u-2}(x+t+1) .
\end{aligned}
$$

Similarly,

$$
\varrho\left(w^{-1}\right)=\left(1+x\left(1+t+t^{2}+\ldots\right)\right)^{-1}=\frac{t+1}{x+t+1} .
$$

Hence for $s, m \geq 0, s+2 m+2 \leq n-1$ and a monomial $p \in \mathbb{Z}_{2}\left[w_{2}, \ldots, w_{n-1}\right]$ of degree $2 m+2+s$ and length $|p|=m+1$ the corresponding element $\left[w^{-1} \cdot \operatorname{Sq} p\right]_{\operatorname{deg}=n}$ 
of $\mathcal{D}$ is mapped by $\varrho$ to

$$
\begin{aligned}
R(s, m):=\varrho\left(\left[w^{-1} \operatorname{Sq} p\right]_{\operatorname{deg}=n}\right) & =\left[\frac{t+1}{x+t+1} x^{m+1} t^{s}(t+1)^{s}(x+t+1)^{m+1}\right]_{\operatorname{deg}=n} \\
& =\left[(t+1)^{s+1} t^{s}(x+t+1)^{m} x^{m+1}\right]_{\operatorname{deg}=n} .
\end{aligned}
$$

Note that if a monomial $p$ is divisible by $w_{1}$, then Sq $p$ and $\left[w^{-1} \operatorname{Sq} p\right]_{\operatorname{deg}=n}$ are also divisible by $w_{1}$. Consequently $\varrho\left(\left[w^{-1} \operatorname{Sq} p\right]_{\operatorname{deg}=n}\right)=0$.

Separating the expression for $R(s, m)$ by degree of $x$ we get

$$
\begin{aligned}
& R(s, m)=\left[\sum_{i=0}^{m}\left(\begin{array}{c}
m \\
i
\end{array}\right) x^{m+1+i} t^{s}(t+1)^{s+1+m-i}\right]_{\mathrm{deg}=n} \\
= & \sum_{i=0}^{m}\left(\begin{array}{c}
m \\
i
\end{array}\right)\left(\begin{array}{c}
s+1+m-i \\
n-2 m-2 i-2-s
\end{array}\right) x^{m+1+i} t^{n-2 m-2 i-2} .
\end{aligned}
$$

Recall that we use the convention that $\left(\begin{array}{l}\alpha \\ \beta\end{array}\right)=0$ if $\beta<0$ or $\alpha<\beta$. Note that the binomial coefficient in the above sum is equal to 0 if the exponent of $t$ is negative. When $p$ is the constant 1 , we have

$$
\begin{aligned}
R_{0} & :=\varrho\left(\left[w^{-1} \mathrm{Sq} 1\right]_{\mathrm{deg}=n}\right)=\left[\frac{t+1}{x+t+1}\right]_{\operatorname{deg}=n}=\left[\frac{1}{1+\frac{x}{1+t}}\right]_{\mathrm{deg}=n} \\
& =\sum_{1 \leq j \leq n / 2} x^{j}\left[(1+t)^{-j}\right]_{\operatorname{deg}=n-2 j}=\sum_{1 \leq j \leq n / 2}\left(\begin{array}{c}
n-j-1 \\
n-2 j
\end{array}\right) x^{j} t^{n-2 j} .
\end{aligned}
$$

Let $V_{R}$ denote the set $\{(s, m): s, m \geq 0, s+2 m+2 \leq n-1\}$. Therefore $\varrho(\mathcal{D})$ is equal to the linear span of the set $\left\{R(s, m):(s, m) \in V_{R}\right\} \cup\left\{R_{0}\right\}$ in $\mathbb{Z}_{2}[x, t]$. Denote the linear span of $\left\{R(s, m):(s, m) \in V_{R}\right\}$ by $\mathcal{R}_{+}$and denote $\varrho(\mathcal{D})$ by $\mathcal{R}$.

4.1.2. The dimension of $\mathcal{R}$. The space $\mathcal{R}$ is contained in $\operatorname{im} \varrho_{n}$, that is, $\mathcal{R}$ is contained in $\left\langle x t^{n-2}, \ldots, x^{\lfloor n / 2\rfloor} t^{n-2\lfloor n / 2\rfloor}\right\rangle$. We will check whether the monomials $x t^{n-2}$, $\ldots, x^{\lfloor n / 2\rfloor} t^{n-2\lfloor n / 2\rfloor}$ are contained in $\mathcal{R}$ separately in the cases of odd and even $n$. We will use the criterion of [G199] cited above, which states that $\left(\begin{array}{l}b \\ a\end{array}\right), 0 \leq a \leq b$, is even if and only if there is a binary position at which $a$ has the digit 1 and $b$ has the digit 0 .

Lemma 4.13. The binomial coefficients $\left(\begin{array}{c}K-p \\ p\end{array}\right)$ with $0<p \leq \frac{K}{2}$ are all even if and only if $K+1$ is a power of 2 .

Proof. If $K+1$ is a power of 2 , then $K$ written in binary form contains only digits 1 , hence $p$ and $K-p$ are complementary to each other. Since $p \neq 0$, there is a digit 1 in its binary representation. Thus in the same position $K-p$ has digit 0 and the criterion of G199] implies that $\left(\begin{array}{c}K-p \\ p\end{array}\right)$ is even. Conversely, if $K$ contains the bit pattern ...10... at position $h$, say, then $\left(\begin{array}{c}K-2^{h} \\ 2^{h}\end{array}\right)$ is odd by the same criterion.

Due to our convention 7 this result implies that $K+1$ is a power of 2 if and only if the binomial coefficients $\left(\begin{array}{c}K-p \\ p\end{array}\right)$ are even for all $p>0$.

${ }^{7}$ If $\beta<0$ or $\alpha<\beta$, then the binomial coefficient $\left(\begin{array}{l}\alpha \\ \beta\end{array}\right)=0$. 
4.1.3. Case of $n$ even. For $n=2$, we have $R_{0}=x$, hence $\operatorname{im} \varrho_{n}=\langle x\rangle=\mathcal{R}$.

For $n>2$, note that the monomial $x t^{n-2}$ occurs as a summand in $R(s, m)$ only in the case $m=0$ and $R(s, 0)=\left(\begin{array}{c}s+1 \\ n-2-s\end{array}\right) x t^{n-2}$. If $n \geq 3$, then for $s=n-3$ we have $0<n-2-s \leq(n-1) / 2$ and $(s, 0) \in V_{R}$. If $n$ is not a power of 2 , then we apply Lemma 4.13 with $K=n-1$ and $p=n-2-s$, and obtain that the coefficient of $x t^{n-2}$ in $R(s, 0)$ is not 0 . If $n$ is a power of 2 , then Lemma 4.13 with the same choice of $K=n-1$ and $p=n-2-s$ implies that $R(s, 0)=0$ for all $(s, 0) \in V_{R}$. Hence $x t^{n-2} \in \mathcal{R}_{+}$if and only if $n$ is not a power of 2 . Note that if $x t^{n-2} \notin \mathcal{R}_{+}$, then $x t^{n-2}$ does not appear as a summand in any elements of $\mathcal{R}_{+}$.

If $n=4$, then $V_{R}=\{(0,0),(1,0)\}$. We have $R(0,0)=0$ as one can check easily, and above we have shown that $R(1,0)=0$. Thus $\mathcal{R}_{+}$consists only of the zero element.

Next consider $m=1,3, \ldots, \frac{n-4}{2}$ for $n \geq 4$ if $4 \nmid n$ and $m=1,3, \ldots, \frac{n-6}{2}$ for $n \geq 6$ if $4 \mid n$. Choosing $s=n-2 m-3$ gives us $(s, m) \in V_{R}$ and $R(n-2 m-3, m)=$ $\left(\begin{array}{c}m \\ 0\end{array}\right)\left(\begin{array}{c}n-m-2 \\ 1\end{array}\right) x^{m+1} t^{n-2 m-2}$. Since $n-m-2$ is odd, $R(n-2 m-3, m)=x^{m+1} t^{n-2 m-2}$. Therefore $x^{m+1} t^{n-2 m-2}$ is in $\mathcal{R}_{+}$. Setting $s=n-2 m-4$ gives $(s, m) \in V_{R}$ and

$$
\begin{aligned}
& R(n-2 m-4, m) \\
& \quad=\left(\begin{array}{c}
m \\
0
\end{array}\right)\left(\begin{array}{c}
n-m-3 \\
2
\end{array}\right) x^{m+1} t^{n-2 m-2}+\left(\begin{array}{c}
m \\
1
\end{array}\right)\left(\begin{array}{c}
n-m-4 \\
0
\end{array}\right) x^{m+2} t^{n-2 m-4} .
\end{aligned}
$$

The first summand is in $\mathcal{R}_{+}$by the argument above, and thus so is the second one, which equals $x^{m+2} t^{n-2 m-4}$ due to $m$ being odd. Therefore we obtain that for $n \geq 4,4 \nmid n$ the monomials $x^{2} t^{n-4}, \ldots, x^{n / 2}$ are in $\mathcal{R}_{+}$, and for $n \geq 6,4 \mid n$ the monomials $x^{2} t^{n-4}, \ldots, x^{(n-2) / 2} t^{2}$ are in $\mathcal{R}_{+}$.

The only monomial not covered by the cases detailed above is $x^{n / 2}$ in the case when $n$ is divisible by 4 and $n \geq 6$. Since all the other monomials either belong to $\mathcal{R}_{+}$or do not appear as summands in any $R(s, m)$ for $(s, m) \in V_{R}$, we have that $x^{n / 2} \in \mathcal{R}_{+}$if and only if $x^{n / 2}$ occurs as a summand in an $R(s, m),(s, m) \in V_{R}$. The coefficient of $x^{n / 2}$ in $R(s, m)$ can be nonzero only when $n=2 m+2 i+2$ for some $0 \leq i \leq m$ and $s=0$, and then the coefficient is

$$
\left(\begin{array}{c}
m \\
i
\end{array}\right)\left(\begin{array}{c}
s+1+m-i \\
n-2 m-2 i-2-s
\end{array}\right)=\left(\begin{array}{c}
m \\
\frac{n}{2}-m-1
\end{array}\right)\left(\begin{array}{c}
2 m+2-\frac{n}{2} \\
0
\end{array}\right)=\left(\begin{array}{c}
m \\
\frac{n}{2}-m-1
\end{array}\right),
$$

and we have $(s, m) \in V_{R}$ if and only if $m \geq i \geq 1, n \geq 6$. By Lemma 4.13, $\left(\begin{array}{c}m \\ \frac{n}{2}-m-1\end{array}\right)$ is even for all possible $m$ exactly when $\frac{n}{2}$ is a power of 2 .

To summarize, when $n$ is even, the set $\left\{R(s, m):(s, m) \in V_{R}\right\}$ generates the space $\operatorname{im} \varrho_{n}=\left\langle x t^{n-2}, \ldots, x^{n / 2}\right\rangle$ if $n$ is not a power of 2 . If $n$ is a power of 2 , then we know that $\mathcal{R}_{+}$is spanned by all but two monomials in im $\varrho$ of degree $n$; the exceptions are $x t^{n-2}$ and $x^{n / 2}$. Let us check the coefficients of $x t^{n-2}$ and $x^{n / 2}$ in $R_{0}=\left(\begin{array}{l}n-2 \\ n-2\end{array}\right) x t^{n-2}+\cdots+\left(\begin{array}{c}n-\frac{n}{2}-1 \\ 0\end{array}\right) x^{n / 2}$. Both of these coefficients are 1 , hence $\mathcal{R}=\left\langle\mathcal{R}_{+} \cup\left\{R_{0}\right\}\right\rangle$ has codimension 1 in $\operatorname{im} \varrho_{n}$ and $\operatorname{im} \varrho_{n} / \mathcal{R}$ is spanned by $x t^{n-2}+\mathcal{R}=x^{n / 2}+\mathcal{R}$.

4.1.4. Case of $n$ odd. Let us call a monomial $x^{h} t^{n-2 h}$ admissible if $h$ is not a power of 2 .

Lemma 4.14. For an admissible monomial $x^{h} t^{n-2 h}$ with $1 \leq h \leq \frac{n-1}{2}$ and $2^{u}<$ $h<2^{u+1}$, where $u \geq 0$, there exist an integer $r(h)$, a set of integers $E_{h}$ with 
$2^{u} \leq \alpha \leq h-1$ for all $\alpha \in E_{h}$, and an element $R_{h} \in \mathcal{R}_{+}$such that $R_{h}+x^{h} t^{n-2 h}$ is a linear combination of monomials $x^{\alpha} t^{n-2 \alpha}, \alpha \in E_{h}$.

Proof. Take the greatest $r=r(h) \geq 0$ for which $2^{r} \mid h$. Note that

$$
h-1, \ldots, h-2^{r} \geq 2^{u}
$$

since $h-2^{r}$ has the same binary form as $h$ except for the least significant digit 1 , which is changed to 0 . Also note that $h \equiv 2^{r} \bmod 2^{r+1}$ and $h \geq 2^{r+1}+2^{r}$. Consider $R\left(n-2 h, h-1-2^{r}\right)$. This polynomial is in $\mathcal{R}_{+}$since $\left(n-2 h, h-1-2^{r}\right) \in V_{R}$. Indeed, $h-1 \geq 2^{r+1}>2^{r}, 2 h<n$ and $n-2 h+2\left(h-1-2^{r}\right)+2=n-2^{r+1}<n$. We have

$$
\begin{aligned}
R\left(n-2 h, h-1-2^{r}\right)=\left(\begin{array}{c}
h-1-2^{r} \\
0
\end{array}\right)\left(\begin{array}{c}
n-h-2^{r} \\
2^{r+1}
\end{array}\right) x^{h-2^{r}} t^{n-2 h+2^{r+1}} \\
+\cdots+\left(\begin{array}{c}
h-1-2^{r} \\
2^{r}
\end{array}\right)\left(\begin{array}{c}
n-h-2^{r+1} \\
0
\end{array}\right) x^{h} t^{n-2 h} .
\end{aligned}
$$

The coefficient of the last monomial of this sum is nonzero because $h \geq 2^{r+1}+2^{r}$, $n-h-2^{r+1}>n-2 h>0$, and $h-2^{r}-1 \equiv-1 \bmod 2^{r+1}$ implies that the $r$-th binary digit of $h-2^{r}-1$ is 1 .

Let $R_{h}$ be $R\left(n-2 h, h-1-2^{r}\right)$ and let $E_{h}$ be $\left\{h-1, \ldots, h-2^{r}\right\}$. Then, by (4.1) we have the statement.

Proposition 4.15. Let $1 \leq h \leq \frac{n-1}{2}$ and assume that $u \geq 0$ is the greatest integer such that $2^{u} \leq h$. Then $x^{h} t^{n-2 h} \in \mathcal{R}_{+}$or $x^{h} t^{n-2 h}+x^{2^{u}} t^{n-2^{u+1}} \in \mathcal{R}_{+}$holds.

Proof. If $h$ is a power of 2 , then the statement obviously holds. Hence we can assume that $x^{h} t^{n-2 h}$ is admissible and $2^{u}<h<2^{u+1}$. Apply Lemma 4.14 to $x^{h} t^{n-2 h}$. Then $x^{h} t^{n-2 h}+R_{h}$ is a linear combination of monomials, where the exponents $\alpha \in E_{h}$ satisfy $2^{u} \leq \alpha \leq h-1$. Let $E_{h}^{\prime}=\left\{\alpha \in E_{h}: \alpha>2^{u}\right\}$.

Again, if $h-1>2^{u}$ and $E_{h}^{\prime} \neq \emptyset$, then apply Lemma 4.14 to the admissible monomials of the linear combination $x^{h} t^{n-2 h}+R_{h}$, and then we obtain that $x^{h} t^{n-2 h}+R_{h}+\sum_{\alpha \in E_{h}^{\prime}} R_{\alpha}$ is a linear combination of monomials whose degree in $x$ is at least $2^{u}$ and smaller than $h-1$. Again, if $h-2>2^{u}$ and there are resulting admissible monomials in the last linear combination, then apply Lemma 4.14 and iterate this procedure until we get that $x^{h} t^{n-2 h}+\tilde{R}=\varepsilon x^{2^{u}} t^{n-2^{u+1}}$, where $\tilde{R} \in \mathcal{R}_{+}$ and $\varepsilon \in\{0,1\}$. Note that the procedure finishes in a finite number of steps since at each step the linear combination of the next step has smaller degrees in $x$, while a common lower limit for the degrees of $x$ is $2^{u}$. This proves our claim.

Proposition 4.16. Let $1 \leq h \leq \frac{n-1}{2}$. For every $r \geq 0$, if $x^{h} t^{n-2 h} \notin \mathcal{R}_{+}$and $n-2 h \geq 2^{r}-1$, then $2^{r} \mid n-h+1$.

Proof. The proof will proceed by induction on $r$, with $r=0$ as the trivial starting case: $1 \mid n-h+1$ always holds.

Let $r \geq 1$ and suppose that the statement holds for $r-1$. Let $h$ be such that $x^{h} t^{n-2 h} \notin \mathcal{R}_{+}$and $n-2 h \geq 2^{r}-1$. Assume indirectly that $2^{r} \nmid n-h+1$. We have $n-2 h \geq 2^{r}-1>2^{r-1}-1$, and hence by the induction hypothesis we have $n-h+1 \equiv 2^{r-1} \bmod 2^{r}$. 
Consider

$$
\begin{aligned}
R\left(n-2 h-2^{r-1}, h-1\right) & =\left(\begin{array}{c}
h-1 \\
0
\end{array}\right)\left(\begin{array}{c}
n-h-2^{r-1} \\
2^{r-1}
\end{array}\right) x^{h} t^{n-2 h} \\
& +\cdots+\left(\begin{array}{c}
h-1 \\
\left\lfloor 2^{r-2}\right\rfloor
\end{array}\right)\left(\begin{array}{c}
n-2 h-2^{r-2} \\
2^{r-1}-2\left\lfloor 2^{r-2}\right\rfloor
\end{array}\right) x^{h+\left\lfloor 2^{r-2}\right\rfloor} t^{n-2 h-2\left\lfloor 2^{r-2}\right\rfloor},
\end{aligned}
$$

where taking the integral part of $2^{r-2}$ is only needed to handle the case of $r=1$. Since $h \geq 1, n-2 h-2^{r-1} \geq 2^{r}-1-2^{r-1}=2^{r-1}-1 \geq 0$ and $n-2 h-2^{r-1}+$ $2(h-1)+2=n-2^{r-1}<n$, we have that $R\left(n-2 h-2^{r-1}, h-1\right) \in \mathcal{R}_{+}$.

For $y=h+1, \ldots, h+\left\lfloor 2^{r-2}\right\rfloor$ we have $n-2 y \geq n-2\left(h+\left\lfloor 2^{r-2}\right\rfloor\right) \geq 2^{r}-1-2^{r-1}=$ $2^{r-1}-1$, and since by the induction hypothesis $2^{r-1} \mid n-h+1$, none of the values $n-y+1$ can be divisible by $2^{r-1}$. Applying the induction hypothesis again gives us that all of the monomials in (4.2) except possibly the first one are in $\mathcal{R}_{+}$. But the coefficient of the first term is nonzero: $\left(\begin{array}{c}h-1 \\ 0\end{array}\right)=1$ and $\left(\begin{array}{c}n-h-2^{r-1} \\ 2^{r-1}\end{array}\right)=1$ by G199. since $n-h-2^{r-1} \equiv 2^{r}-1 \bmod 2^{r}$ has the binary digit 1 at the only location where $2^{r-1}$ has a 1 . Therefore $R\left(n-2 h-2^{r-1}, h-1\right)+x^{h} t^{n-2 h} \in \mathcal{R}_{+}$and consequently $x^{h} y^{n-2 h} \in \mathcal{R}_{+}$, finishing the proof.

We apply Proposition 4.16 to monomials of the form $x^{2^{u}} t^{n-2^{u+1}}, u \geq 0, n$ is odd and $2^{u+1} \leq n$, with the choice of $r \geq 0$ so that $2^{r+1}<n<2^{r+2}$. Note that $u \leq r$ due to $2^{u} \leq \frac{n-1}{2}<2^{r+1}$. We get that if $x^{2^{u}} t^{n-2^{u+1}} \notin \mathcal{R}_{+}$, then at least one of the following has to hold:

(a) $2^{r} \mid n-2^{u}+1$. We know that $n-2^{u}$ is at least $n-2^{r}>2^{r+1}-2^{r}=2^{r}$, and on the other hand $n-2^{u}$ is at most $n-1<2^{r+2}-1$. There are only two integers $i$ in the open interval $\left(2^{r}, 2^{r+2}-1\right)$ which satisfy the divisibility condition $2^{r} \mid i+1$, namely $2^{r+1}-1$ and $3 \cdot 2^{r}-1$. Hence $n-2^{u}$ is either $2^{r+1}-1$ or $3 \cdot 2^{r}-1$.

(b) $n-2^{u+1}<2^{r}-1$. Then $2^{u+1}>n-2^{r}+1>2^{r}$ since $2^{r+1}<n$, and $u \leq r$ implies that $u=r$.

We claim that in the case $n-2^{u}=3 \cdot 2^{r}-1$ of (a) the monomial $x^{2^{u}} t^{n-2^{u+1}}$ is actually in $\mathcal{R}_{+}$. Indeed, check the statement of Proposition 4.16 for $r+1$. Then $2^{r+1} \nmid n-2^{u}+1=3 \cdot 2^{r}$, and $n-2^{u+1}=3 \cdot 2^{r}-1-2^{u} \geq 2^{r+1}-1$ since $2^{u} \leq 2^{r}$. Therefore we have $x^{2^{u}} t^{n-2^{u+1}} \in \mathcal{R}_{+}$.

Hence if $x^{2^{u}} t^{n-2^{u+1}} \notin \mathcal{R}$, then we are left with two possibilities:

(a) $n=2^{r+1}+2^{u}-1$,

(b) $n-2^{u+1}<2^{u}-1$ and $2^{u+1}<n<2^{u+2}$.

In case (b), note that if $x^{2^{u}} t^{n-2^{u+1}} \notin \mathcal{R}_{+}$, then Proposition 4.16 implies that for any $0 \leq r^{\prime} \leq u$ either

(i) $n-2^{u+1}<2^{r^{\prime}}-1$ or

(ii) $n-2^{u} \equiv 2^{r^{\prime}}-1 \bmod 2^{r^{\prime}}$. Due to $r^{\prime} \leq u$ this condition is equivalent to $n-2^{u+1} \equiv 2^{r^{\prime}}-1 \bmod 2^{r^{\prime}}$.

In case (b) choose $r^{\prime}$ to satisfy $2^{r^{\prime}} \leq n-2^{u+1}<2^{r^{\prime}+1}$. This value of $r^{\prime}$ will be smaller than $u$ due to $n-2^{u+1}<2^{u}-1$. For this choice of $r^{\prime}$, condition (i) fails; thus condition (ii) has to hold. This implies that $n-2^{u+1}-\left(2^{r^{\prime}}-1\right)=l 2^{r^{\prime}}$. This integer $l$ can be only 1 because $n-2^{u+1}<2^{r^{\prime}+1}$. Thus, $n-2^{u+1}=2^{r^{\prime}+1}-1$. 
Therefore if $x^{2^{u}} t^{n-2^{u+1}} \notin \mathcal{R}_{+}$, then we have two possible cases:

(a) $n=2^{r+1}+2^{u}-1$, where we chose $r \geq 0$ so that $2^{r+1}<n<2^{r+2}$; this implied $u \leq r$,

(b) $n=2^{u+1}+2^{r^{\prime}+1}-1$, where we chose $r^{\prime} \geq 0$ so that $2^{r^{\prime}} \leq n-2^{u+1}<2^{r^{\prime}+1}$; this implied $r^{\prime}<u$.

By Proposition 4.15 in both cases (a) and (b) we have $\mathcal{R}_{+}=\operatorname{im} \varrho_{n}$, unless there are positive integers $a>b$ such that $n=2^{a}+2^{b}-1$. Moreover, in these exceptional cases, when $n=2^{a}+2^{b}-1$ with $a>b>0$, the linear space $\mathcal{R}_{+}$has to contain all the monomials $x^{2^{u}} t^{n-2^{u+1}}$ except possibly those with $u=a-1$ (in case (b)) and $u=b($ in case $(\mathrm{a}))$.

Proposition 4.17. If $n=2^{a}+2^{b}-1$ with $a>b>0$ and $u=a-1$ or $u=b$, then the monomial $x^{2^{u}} t^{n-2^{u+1}}$ does not appear as a summand in any $R(s, m) \in \mathcal{R}_{+}$.

Proof. In the relation $R(s, m)$ the monomial $x^{2^{u}} t^{n-2^{u+1}}$ has the coefficient

$$
\begin{aligned}
\left(\begin{array}{c}
m \\
2^{u}-m-1
\end{array}\right)\left(\begin{array}{c}
m+s+1-\left(2^{u}-m-1\right) \\
n-2 m-2\left(2^{u}-m-1\right)-2-s
\end{array}\right) & =\left(\begin{array}{c}
m \\
2^{u}-1-m
\end{array}\right)\left(\begin{array}{c}
2 m+2-2^{u}+s \\
n-2^{u+1}-s
\end{array}\right) .
\end{aligned}
$$

The first binomial coefficient is even unless $2^{u}=m+1$ according to Lemma 4.13 for $K=2^{u}-1$. Hence we only consider the case $m=2^{u}-1$. Then the second binomial coefficient becomes $\left(\begin{array}{c}2^{u}+s \\ n-2^{u+1}-s\end{array}\right)$ with $s$ running from 0 to $n-2 m-3=n-2^{u+1}-1$ as follows from the condition $(s, m) \in V_{R}$. For $u=b$ we have $n-2^{u}=2^{a}-1$, and Lemma 4.13 with $K=n-2^{u}$ implies that all of these coefficients (4.3) are even. For $u=a-1$ we have $2^{u+1}+s \geq 2^{u+1}=2^{a}$ and $n-2^{u+1}-s \leq n-2^{a}=$ $2^{b}-1<2^{a-1}$. Thus the criterion of [G199] gives the same results for $\left(\begin{array}{c}2^{u}+s \\ n-2^{u+1}-s\end{array}\right)$ and $\left(\begin{array}{c}2^{u}+s-2^{a-1} \\ n-2^{u+1}-s\end{array}\right)=\left(\begin{array}{c}s \\ 2^{b}-1-s\end{array}\right)$. Since $n-2^{u+1}-s \geq 1$, Lemma 4.13 proves that $\left(\begin{array}{c}s \\ 2^{b}-1-s\end{array}\right)$ is even for all choices of $s$, as claimed.

This means that if $n=2^{a}+2^{b}-1, a>b>0$, then the monomials $x^{2^{a-1}} t^{n-2^{a}}$ and $x^{2^{b}} t^{n-2^{b+1}}$ never appear as summands in any $R(s, m) \in \mathcal{R}_{+}$and hence the algorithm of Proposition 4.15 leads to $x^{h} t^{n-2 h} \in \mathcal{R}_{+}$when $u=a-1$ or $u=$ $b$. Consequently, $\mathcal{R}_{+}$is spanned by all monomials from $x t^{n-2}$ to $x^{\frac{n-1}{2}} t$ except $x^{2^{a-1}} t^{n-2^{a}}$ and $x^{2^{b}} t^{n-2^{b+1}}$, which span a linear space complementary to $\mathcal{R}_{+}$.

To summarize, when $n$ is odd, then we have three possibilities:

- If $n \neq 2^{a}+2^{b}-1$ for any $a>b>0$, then $\mathcal{R}_{+}=\operatorname{im} \varrho_{n}$.

- If $n=3 \cdot 2^{b}-1$ for some $b>0$, then

$$
\mathcal{R}_{+}=\left\langle x t^{n-2}, \ldots, x^{2^{b}-1} t^{n-2^{b+1}+2}, x^{2^{b}+1} t^{n-2^{b+1}-2}, \ldots, x^{\frac{n-1}{2}} t\right\rangle .
$$

- If $n=2^{a}+2^{b}-1$ for some $a>b+1, b>0$, then

$$
\begin{aligned}
\mathcal{R}_{+}=\left\langle x t^{n-2}\right. & , \ldots, x^{2^{b}-1} t^{n-2^{b+1}+2}, x^{2^{b}+1} t^{n-2^{b+1}-2} \\
& \left.\ldots, x^{2^{a-1}-1} t^{n-2^{a}+2}, x^{2^{a-1}+1} t^{n-2^{a}-2}, \ldots, x^{\frac{n-1}{2}} t\right\rangle .
\end{aligned}
$$

Finally, the relation $R_{0}$ contains the monomials $x^{2^{a-1}} t^{n-2^{a}}$ and $x^{2^{b}} t^{n-2^{b+1}}$ with the coefficients $\left(\begin{array}{c}n-2^{a-1}-1 \\ n-2^{a}\end{array}\right)=\left(\begin{array}{c}2^{b}+2^{a-1}-2 \\ 2^{b}-1\end{array}\right)$ and $\left(\begin{array}{c}n-2^{b}-1 \\ n-2^{b+1}\end{array}\right)=\left(\begin{array}{c}2^{a}-2 \\ 2^{a}-2^{b}-1\end{array}\right)$, both of which are

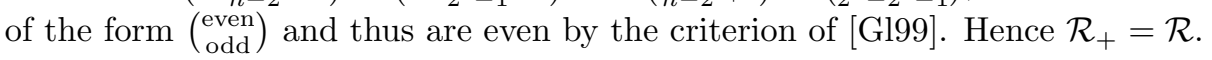


4.1.5. Proofs of the statements. We define linear subspaces of $\mathfrak{N}_{n}$ as follows.

Definition 4.18. - For $n=2^{a}$ with $a \geq 2, \mathfrak{A}^{1}$ is the 1-dimensional space defined by vanishing of $w_{2}^{n / 2}+w_{n}$ as well as all monomial Stiefel-Whitney numbers except $w_{2}^{n / 2}$ and $w_{n}$. For example, the cobordism class of $\left(\mathbb{C} P^{2}\right)^{n / 4}$ generates $\mathfrak{A}^{1}$.

- For $n=2^{b+1}+2^{b}-1$ with $b \geq 1, \mathfrak{B}^{1}$ is the 1 -dimensional space defined by the vanishing of

- all monomial Stiefel-Whitney numbers not of the form $w_{m_{1}} \cdots w_{m_{2 b}}$,

- all monomial Stiefel-Whitney numbers containing $w_{1}$,

- all pairwise sums of the rest of the monomial Stiefel-Whitney numbers.

- For $n=2^{a}+2^{b}-1$ with $a \geq b+2$ and $b \geq 1, \mathfrak{C}^{2}$ is the 2-dimensional space defined by the vanishing of

- all monomial Stiefel-Whitney numbers which are not either of the form

$$
w_{m_{1}} \cdots w_{m_{2^{b}}} \text { or } \quad w_{m_{1}} \cdots w_{m_{2^{a-1}}}
$$

- all monomial Stiefel-Whitney numbers containing $w_{1}$,

- all pairwise sums of Stiefel-Whitney numbers of the form $w_{m_{1}} \cdots w_{m_{2^{a-1}}}$ with all $m_{j} \geq 2$, and all pairwise sums of Stiefel-Whitney numbers of the form $w_{m_{1}} \cdots w_{m_{2^{b}}}$ with all $m_{j} \geq 2$.

Proof of Theorem 1.6. By the above, if $n \neq 2^{a}+2^{b}-1, a>b \geq 0$, then any oriented $n$-manifold which has a fold map in codimension -1 is unoriented null-cobordant. Unless $n$ is divisible by 4 , this implies that $M$ is also oriented null-cobordant; see Wa60.

In the case of $n=2^{a}+2^{b}-1, a>b \geq 0$, the Stiefel-Whitney characteristic numbers of $M$ which belong to the complete preimage $\varrho^{-1}(\mathcal{R})$ have to vanish. This leaves the following possibilities for nonzero characteristic numbers:

- If $n$ is a power of 2 , then $\varrho^{-1}(\mathcal{R})$ is spanned by $w_{n}+w_{2}^{n / 2}$ and all monomials except $w_{n}$ and $w_{2}^{n / 2}$. In this case $[M] \in \mathfrak{A}^{1}$.

- If $n=2^{a}+2^{b}-1, a>b>0$, then $\varrho^{-1}(\mathcal{R})$ is spanned by all monomials of length not equal to either $2^{b}$ or $2^{a-1}$ as well as the relations in $\mathcal{I}$ corresponding to these exceptional lengths. When $a=b+1$, the two lengths coincide and we get that $[M] \in \mathfrak{B}^{1}$, while in the other case we get that $[M] \in \mathfrak{C}^{2}$.

In the remaining case of $n$ divisible by 4 , we need to additionally calculate the Pontryagin characteristic numbers of $M$ to determine its oriented cobordism class. Theorem 1.4] shows that all the rational Pontryagin classes of $M$ except $p_{1}^{\mathbb{Q}}(M)$ have to vanish. Hence the only Pontryagin number that may be nonzero is $p_{1}^{n / 4}[M]$. If we additionally assume that $M$ is unoriented null-cobordant, then this number has to be even, as its reduction modulo 2 is the Stiefel-Whitney characteristic number $w_{2}^{n / 2}[M]$.

Proof of Theorem 1.8. By Proposition 3.1 we know that $T M \oplus \varepsilon^{1}=\zeta^{2} \oplus \varepsilon^{n-1}$ for some 2-dimensional bundle $\zeta$. Hence $w(M)=1+w_{1}(\zeta)+w_{2}(\zeta)$, and we will denote the characteristic class $w_{i}(\zeta)$ by $w_{i}$ for brevity. The only nonzero total Steenrod squares of these classes are $S q\left(w_{1}\right)=w_{1}\left(1+w_{1}\right)$ and $S q\left(w_{2}\right)=$ $w_{2}+w_{2} w_{1}+w_{3}+w_{2}^{2}=w_{2}\left(1+w_{1}+w_{2}\right)$. Thus, we can compute the Dold relation 
corresponding to the polynomial $w_{1}^{a} w_{2}^{b}$ with $a+2 b \leq n-1, a, b \geq 0$, as the degree $n$ part of the expression

$$
\frac{w_{1}^{a}\left(1+w_{1}\right)^{a} w_{2}^{b}\left(1+w_{1}+w_{2}\right)^{b}}{1+w_{1}+w_{2}}=w_{1}^{a}\left(1+w_{1}\right)^{a} w_{2}^{b}\left(1+w_{1}+w_{2}\right)^{b-1} .
$$

Setting $b=0,0 \leq a \leq n-1$ gives

$$
\begin{gathered}
R(a):=\left[\frac{w_{1}^{a}\left(1+w_{1}\right)^{a}}{1+w_{1}+w_{2}}\right]_{\mathrm{deg}=n}=\left[\frac{w_{1}^{a}\left(1+w_{1}\right)^{a-1}}{1+\frac{w_{2}}{1+w_{1}}}\right]_{\mathrm{deg}=n} \\
=\left[w_{1}^{a}\left(1+w_{1}\right)^{a-1} \sum_{j=0}^{\infty} \frac{w_{2}^{j}}{\left(1+w_{1}\right)^{j}}\right]_{\mathrm{deg}=n}^{\frac{n-a}{2}}=\sum_{j=0}^{w_{1}^{a}} w_{2}^{j}\left[\left(1+w_{1}\right)^{a-1-j}\right]_{\operatorname{deg}=n-a-2 j} \\
=\sum_{j=0}^{\frac{n-a}{2}}\left(\begin{array}{c}
a-1-j \\
n-a-2 j
\end{array}\right) w_{1}^{n-2 j} w_{2}^{j} .
\end{gathered}
$$

Here we use the analytical definition of binomial coefficients: $\left(\begin{array}{l}u \\ v\end{array}\right)=0$ if $v<0$, $\left(\begin{array}{l}u \\ 0\end{array}\right)=1$ and $\left(\begin{array}{l}u \\ v\end{array}\right)=\frac{u(u-1) \cdots(u-v+1)}{v !}$ in the other cases. Choosing $a=n-2 m$ for any $0<m \leq n / 2$ we get

$$
\begin{aligned}
R(n-2 m) & =\sum_{j=0}^{m}\left(\begin{array}{c}
n-2 m-1-j \\
2 m-2 j
\end{array}\right) w_{1}^{n-2 j} w_{2}^{j} \\
& =\left(\begin{array}{c}
n-2 m-1 \\
2 m
\end{array}\right) w_{1}^{n}+\cdots+\left(\begin{array}{c}
n-3 m-1 \\
0
\end{array}\right) w_{1}^{n-2 m} w_{2}^{m}
\end{aligned}
$$

with analytical binomial coefficients. Here the exponent of $w_{2}$ in all the summands except the last one is less than $m$, and the last summand has coefficient 1. Therefore for all $0<m \leq n / 2$ the monomial $w_{2}^{m} w_{1}^{n-2 m}$ is linearly dependent on the monomials with smaller exponents of $w_{2}$ and $R(n-2 m)$. Consequently, all the monomials $w_{2} w_{1}^{n-2}, \ldots, w_{2}^{m} w_{1}^{n-2 m}$ are linearly dependent on $w_{1}^{n}$ and $\{R(n-2 m): 1 \leq m \leq n / 2\}$. Evaluating these classes on the fundamental class of $M$, we get that all Stiefel-Whitney characteristic numbers of $[M]$ depend linearly on $w_{1}^{n}[M]$ (with coefficients depending only on $n$ ). This condition either defines the 1-dimensional linear subspace $\mathfrak{D}^{1} \leq \mathfrak{N}_{n}$ or implies that $M$ is unoriented null-cobordant.

Proof of Proposition 1.9. Choose any index set $I=\left(i_{1}, \ldots, i_{r}\right)$ such that $r=|I| \geq$ 2 and $\sum_{j=1}^{r} i_{j}=n$. If for any $j$ we have $i_{j} \leq k$, then $w_{I}=0$ due to the vanishing condition imposed on the Stiefel-Whitney classes of $T M$. If $i_{j} \geq k+1$ for $j=$ $1, \ldots, r$, then for $J=(n-(r-1)(k+1), k+1, \ldots, k+1)$ the characteristic numbers $w_{I}[M]$ and $w_{J}[M]$ coincide by Corollary 4.12, But $w_{k+1}(M)=0$ by the solution to $\left[\right.$ MS74, Problem 8-B], implying that $w_{J}[M]=0$ and thus $w_{I}[M]=0$ whenever $|I| \geq 2$.

Proof of Proposition 1.10, Perturb the Morin map to get a cusp map Sad03. By Proposition 4.11 and the solution to [MS74, Problem 8-B], the statement follows similarly to the previous one; details are left to the reader. 


\section{ACKNOWLEDGEMENT}

We thank András Szücs for advice which improved the paper.

\section{REFERENCES}

[An85] Y. Ando, On the elimination of Morin singularities, J. Math. Soc. Japan 37 (1985), no. 3, 471-487. MR792988 (87h:58018)

[An87] , On the higher Thom polynomials of Morin singularities, Publ. RIMS, Kyoto Univ. 23 (1987), 195-207. MR792988 (87h:58018)

[An01] , Folding maps and the surgery theory on manifolds, J. Math. Soc. Japan 53 (2001), no. 2, 357-382. MR1815139 (2002c:57056)

[An04] Existence theorems of fold maps, Japan J. Math. 30 (2004), 29-73. MR.1815139 (2002c:57056)

[An07] , A homotopy principle for maps with prescribed Thom-Boardman singularities, Trans. Amer. Math. Soc. 359 (2007), 489-515. MR2255183 (2007j:58046)

[At61] M. F. Atiyah, Immersions and embeddings of manifolds, Topology 1 (1961), 125-132. MR 0145549(26:3080)

[BH04] A. Banyaga and D. Hurtubise, A proof of the Morse-Bott lemma, Expo. Math. 22 (2004), 365-373. MR2075744(2005b:57062)

[Boa67] J. M. Boardman, Singularities of differentiable maps, IHES Publ. Math. 33 (1967), 21-57. MR 0231390(37:6945)

[Ch83] D. S. Chess, A note on the classes $\left[S_{1}^{k}(f)\right]$, Proc. Symp. Pure Math. 40 (1983), 221-224. MR.713061 (85f:57020)

[Do56] A. Dold, Erzeugende der Thomschen Algebra N, Math. Z. 65 (1956), 25-35. MR0079269 (18:60c)

[Ga78] A. M. Gabrielov, Combinatorial formulas for Pontrjagin classes and GL-invariant chains. (Russian) Funktsional. Anal. i Prilozhen. 12 (1978), no. 2, 1-7, 95. MR 498893 (80b:57018)

[G199] J. W. L. Glaisher, On the residue of a binomial-theorem coefficient with respect to a prime modulus, Quart. J. Pure App. Math. 30 (1899), 150-156.

[GG73] M. Golubitsky and V. Guillemin, Stable mappings and their singularities, Graduate Texts in Math. 14, Springer-Verlag, New York, 1973. MR0341518 (49:6269)

[GP07] H. Geiges and F. Pasquotto, A formula for the Chern classes of symplectic blow-ups, J. London Math. Soc. 76 (2007), 313-330. MR2363418 (2008j:53143)

[Mi97] G. Mikhalkin, Blowup equivalence of smooth closed manifolds, Topology 36 (1997), 287299. MR1410476 (98f:57046)

[MS74] J. Milnor and J. D. Stasheff, Characteristic classes, Ann. of Math. Studies, No. 76, Princeton Univ. Press, Princeton, N. J.; Univ. of Tokyo Press, Tokyo, 1974. MR0440554 $(55: 13428)$

[OSS03] T. Ohmoto, O. Saeki and K. Sakuma, Self-intersection class for singularities and its application to fold maps, Trans. Amer. Math. Soc. 355 (2003), 3825-3838. MR 1990176 (2004g:57047)

[Pr08] P. Pragacz, Thom polynomials and Schur functions: Toward the singularities $A_{i}(-)$, Real and complex singularities, Contemporary Mathematics 459 (2008), 165-178. MR2444400 (2010b:58058)

[Roh52] V. A. Rohlin, Intrinsic definition of Pontryagin's characteristic cycles. (Russian) Doklady Akad. Nauk SSSR (N.S.) 84 (1952), 449-452. MR0050276 (14:306c)

[Ron71] F. Ronga, Le calcul de la classe de cohomologie entire duale $\bar{\sum}^{k}$. (French) Proceedings of Liverpool Singularities-Symposium, I (1969/70), pp. 313-315. Lecture Notes in Math., Vol. 192, Springer, Berlin, 1971. MR0293648 (45:2725)

[Ron72] _ Le calcul des classes duales aux singularités de Boardman dordre deux, Comm. Math. Helv. 47 (1972), 15-35. MR0309129 (46:8240)

[Sad03] R. Sadykov, The Chess conjecture, Alg. Geom. Topol. 3 (2003), 777-789. MR1997337 (2005a:57027)

[SSS10] R. Sadykov, O. Saeki and K. Sakuma, Obstructions to the existence of fold maps, J. Lond. Math. Soc. (2) 81 (2010), no. 2, 338-354. MR2602999(2011b:57034) 
[Sae92] O. Saeki, Notes on the topology of folds, J. Math. Soc. Japan 44 (1992), 551-566. MR.1167382 (93f:57037)

[SS98] O. Saeki and K. Sakuma, Maps with only Morin singularities and the Hopf invariant one problem, Math. Proc. Camb. Phil. Soc. 124 (1998), 501-511. MR:1636580 (99h:57058)

[Wa60] C. T. C. Wall, Determination of the cobordism ring, Ann. Math. 72 (1960), 292-311. MR0120654 (22:11403)

Alfréd Rényi Institute of Mathematics, Hungarian Academy of Sciences, Reáltanoda U. 13-15, 1053 Budapest, Hungary

E-mail address: kalmar.boldizsar@renyi.mta.hu

Alfréd Rényi Institute of Mathematics, Hungarian Academy of Sciences, Reáltanoda U. 13-15, 1053 Budapest, Hungary

E-mail address: terpai@math.elte.hu 